\title{
THE GLOBAL DYNAMICS OF DISCRETE SEMILINEAR PARABOLIC EQUATIONS*
}

\author{
C. M. ELLIOTT ${ }^{\dagger}$ AND A. M. STUART ${ }^{\ddagger}$
}

\begin{abstract}
A class of scalar semilinear parabolic equations possessing absorbing sets, a Lyapunov functional, and a global attractor are considered. The gradient structure of the problem implies that, provided all steady states are isolated, solutions approach a steady state as $t \rightarrow \infty$. The dynamical properties of various finite difference and finite element schemes for the equations are analysed. The existence of absorbing sets, bounded independently of the mesh size, is proved for the numerical methods. Discrete Lyapunov functions are constructed to show that, under appropriate conditions on the mesh parameters, numerical orbits approach steady state solutions as discrete time increases. However, it is shown that insufficient spatial resolution can introduce deceptively smooth spurious steady solutions and cause the stability properties of the true steady solutions to be incorrectly represented. Furthermore, it is also shown that the explicit Euler scheme introduces spurious solutions with period 2 in the timestep. As a result, the absorbing set is destroyed and there is initial data leading to blow up of the scheme, however small the mesh parameters are taken. To obtain stabilization to a steady state for this scheme, it is necessary to restrict the timestep in terms of the initial data and the space step. Implicit schemes are constructed for which absorbing sets and Lyapunov functions exist under restrictions on the timestep that are independent of initial data and of the space step; both one-step and multistep (BDF) methods are studied.
\end{abstract}

Key words. attractors, absorbing sets, Lyapunov functions, spurious solutions

AMS subject classifications. $65 \mathrm{M} 99,35 \mathrm{~K} 57$

1. Introduction. In this paper we consider the following semilinear parabolic initial value problem:

$$
\begin{gathered}
u_{t}=\gamma \Delta u-f(u), \quad x \in \Omega, \quad t>0, \\
u=0, \quad x \in \partial \Omega, \quad t \geq 0, \\
u(x, 0)=u_{0}(x), \quad x \in \Omega .
\end{gathered}
$$

Here $\Omega$ is a bounded domain in $\mathbb{R}^{d}(d \leq 3)$ with a Lipschitz boundary $\partial \Omega$ and $\gamma>0$. We shall assume that the initial data $u_{0} \in K_{i}, i=1$ or 2 where $K_{1}=\left\{\eta \in L^{2}(\Omega)\right\}$ and $K_{2}=\left\{\eta \in L^{2}(\Omega):\|\eta\|_{\infty} \leq M\right\}$. The assumptions on the nonlinear function $f(u)$ are:

(F1) $f(\bullet) \in C^{2}(\mathbb{R}, \mathbb{R})$ and $f(0)=0$.

(F2) There exists $\bar{u}>0$ such that $f(r) / r>0$ for any $r:|r|>\bar{u}$.

(F3) $F^{\prime \prime}(u) \geq-C_{F}$, where

$$
F(u)=\int f(u) d u
$$

* Received by the editors April 14, 1992; accepted for publication (in revised form) December 28, 1992.

† School of Mathematical and Physical Sciences, University of Sussex, Brighton BN1 9QH, United Kingdom.

$\ddagger$ School of Mathematical Sciences, University of Bath, Bath BA2 7AY, United Kingdom. Current address, Program in Scientific Computing and Computational Mathematics, Division of Applied Mechanics, Durand 252, Stanford University, California 94305-4040. 
Assumptions (F1), (F2), and (F3) are made throughout the paper unless otherwise stated. Under these assumptions the following global existence result may be proved. For initial data in $K_{1}$ this result requires an additional growth assumption such as

$$
|f(r)| \leq c_{0}|r|^{2 p-1}+c_{1}
$$

(see [26]), whereas for initial data in $K_{2}$ the result utilises the theory of invariant regions (see [28]).

(R1) For each $u_{0} \in K_{i}$ there exists a unique solution of (1.1)-(1.3) which satisfies, for all $T>0$,

$$
u \in L^{2}\left(0, T ; H_{0}^{1}(\Omega)\right) \cap C\left[0, T ; L^{2}(\Omega)\right]
$$

and the mapping $u_{0} \rightarrow u(t)$ is continuous for each $t>0$. Hence the family of solution operators $\{S(t)\}_{t \geq 0}$ defined by $S(t) u_{0} \equiv u(t)$ forms a continuous semigroup on $L^{2}(\Omega)$. In addition, if $u_{0} \in H_{0}^{1}(\Omega) \cap K_{i}$ then for all $T>0$

$$
u \in C\left([0, T) ; H_{0}^{1}(\Omega)\right) \cap L^{2}\left(0, T ; H^{2}(\Omega)\right) .
$$

It is possible to show the following results about the semigroup $S(t)$ (see [11], [8], [19], [27], [26]):

(R2) There exist balls $B_{1}=\left\{v \in L^{2}(\Omega):|v| \leq \rho_{1}\right\}$ and $B_{2}=\left\{v \in H_{0}^{1}(\Omega)\right.$ : $\left.\|v\| \leq \rho_{2}\right\}$, which are absorbing sets for the semigroup $\{S(t)\}_{t \geq 0}$; that is there exist $t_{1}$ and $t_{2}$ depending on $\left\{\left|u_{0}\right|, \rho_{1}\right\}$ and $\left\{\left|u_{0}\right|, \rho_{2}\right\}$, respectively, such that $S(t) u_{0} \in B_{i}$ for all $t \geq t_{i}(i=1,2)$. Here $|\bullet|$ and $\|\bullet\|$ denote the standard $L^{2}$ and $H_{0}^{1}$ norms, respectively.

(R3) There exists a global attractor $\mathcal{A} \subset L^{2}(\Omega)$ for the semigroup $\{S(t)\}_{t>0}$; that is, $\mathcal{A}$ attracts the bounded sets of $L^{2}(\Omega)$, and $\mathcal{A}$ is bounded in $H_{0}^{1}(\Omega) \cup L^{\infty}(\Omega)$ and compact and connected in $L^{2}(\Omega)$.

(R4) The functional

$$
I(v):=\int_{\Omega}\left[\frac{\gamma}{2}|\nabla v(x)|^{2}+F(v(x))\right] d x
$$

where $F(u)$ is defined by (1.4), is a Lyapunov functional for $\{S(t)\}_{t \geq 0}$, that is,

$\mathrm{I}(\mathrm{i})$ There exists $c_{l}$ such that $I(v) \geq c_{l}$ for all $v \in H_{0}^{1}(\Omega)$, and for every $c$ there exists $\rho=\rho(c)$ such that the diameter of $\{v: I(v) \leq c\}$ is bounded by $\rho$.

I(ii) $I(S(t) v) \leq I\left(S\left(t^{\prime}\right) v\right)$ and for all $t \geq t^{\prime}>0$ and for all $v \in H_{0}^{1}(\Omega)$.

I(iii) If $I(S(t) v)=I(v)$ for all $t>0$ then $v \in \mathcal{E}$ where $\mathcal{E}$ is the set of equilibrium points $v$ satisfying

$$
-\gamma \Delta v+f(v)=0, \quad x \in \Omega, \quad v=0, \quad x \in \partial \Omega .
$$

Furthermore, for any $u_{0} \in L^{2}(\Omega)$ the $\omega$-limit set

$$
\omega\left(u_{0}\right)=\left\{v \in L^{2}(\Omega): \exists t_{n} \rightarrow \infty: \lim _{n \rightarrow \infty} S\left(t_{n}\right) u_{0}=v\right\}
$$

is contained in the set of equilibrium solutions (1.6), denoted $\mathcal{E}$, and, if the equilibria are isolated, then

$$
\mathcal{A}=\bigcup_{v \in \mathcal{E}} W^{u}(v)
$$


where $W^{u}(v)$ denotes the unstable manifold of $v$.

It is our purpose in this paper to consider discrete dynamical systems generated by temporal and spatial discretisation of (1.1). We study the existence of absorbing sets, Lyapunov functions and attractors for the approximations and, in addition, describe various spurious features introduced by both spatial and temporal discretisation. We suppose that semidiscretisation in space yields a $J$-dimensional system of ordinary differential equations in time of the form

$$
\begin{gathered}
M \frac{d \mathbf{U}}{d t}+\gamma A \mathbf{U}+M \mathbf{f}(\mathbf{U})=0, \quad t>0, \\
\mathbf{U}(0)=\mathbf{U}_{0} .
\end{gathered}
$$

Here $\mathbf{U}=\left(U_{1}, \ldots, U_{J}\right)^{T}$ and $\{\mathbf{f}(\mathbf{U})\}_{i}=f\left(U_{i}\right)$. We use the notation that, for any $\phi$ : $\mathbb{R} \rightarrow \mathbb{R}$

$$
\{\phi(\mathbf{U})\}_{\mathbf{i}}=\phi\left(\mathbf{U}_{\mathbf{i}}\right)
$$

$M$ is a diagonal matrix with positive entries and $A$ is a symmetric positive definite matrix.

If $\Omega=(0,1)^{d}$ then such a system arises from finite difference approximations on a uniform grid $\{(i h, j h, k h): i, j, k \in[0, N], h=1 / N\}$ with the elements of $M$ being $h^{d}$ and $1 / h^{d} A$ being the matrix arising from a central difference approximation to $-\Delta$. The system also arises from a finite element approximation based on piecewise linear functions on a triangulation of $\Omega$. Denoting by $\left\{\psi_{i}\right\}_{i=1}^{N_{V}}$ the set of piecewise linear basis functions associated with the internal vertices of the elements, then

$$
M_{i i}=\left\langle\psi_{i}, 1\right\rangle^{h}, \quad A_{i j}=\left\langle\nabla \psi_{i}, \nabla \psi_{j}\right\rangle^{h},
$$

where $\langle., .\rangle^{h}$ denotes a discrete $L^{2}$ inner product defined by lumped mass integration.

Section 2 contains various general results on dissipative dynamical systems which will be required throughout the paper. In $\S 3$ we analyse the semidiscrete problem (1.8)-(1.9). Semidiscrete analogues of (R1)-(R4) are proved. However, it is also shown that insufficient spatial resolution can lead to the existence of deceptively smooth spurious steady solutions and also to incorrect stability properties for the true steady states-there exist many more discrete stable steady states than true stable steady states. In $\S 4$ we study the spurious dynamics of the explicit Euler scheme. It is shown that the method can produce period 2 solutions in $n$, the timestep, for arbitrarily small $\Delta t$. Furthermore it is shown that, however small $\Delta t$ and $\Delta x$ are taken, the scheme blows up with appropriate choice of initial data. Thus a global attractor cannot exist for the Euler method considered as a dynamical system on the whole of $\mathbb{R}^{J}$. We derive a restriction on the timestep in terms of both the spatial grid and the magnitude of the initial data, under which discrete analogues of (R1)-(R4) hold. In $\S 5$, various implicit schemes are considered. Discrete analogues of (R1)-(R4) are proved under far less restrictive conditions on the timestep than for the explicit Euler scheme; in particular schemes are constructed for which the timestep restriction is independent of initial data and of $h$. Section 6 is concerned with multistep backward differentiation formulae and similar results to those in $\S 5$ are proved. Finally, in $\S 7$, numerical results are presented to illustrate the theory.

Similar questions to those addressed in this paper have been discussed for spectral approximations of (1.1) in [22] and for finite difference approximations to the 
Kuramoto-Sivashinsky equation in [6]. For Runge-Kutta methods applied to ordinary differential equations, see [14]. The question of convergence of attractors and of attracting sets for semilinear evolution equations is discussed in, for example, [9], [10], and [16]. The question of spurious steady states for such problems has been simultaneously studied in [1]. The treatment of discrete Lyapunov functionals, which are used extensively in this paper, is motivated by studies of the Cahn-Hilliard equation [5].

2. Dissipative dynamical systems. In this section we review the theory of dissipative dynamical systems sufficient for our needs. It is convenient to consider discrete and continuous dynamical systems simultaneously. Let $H$ be a complete metric space and $t$ be a real parameter taking values in $\mathbb{R}$ or $\mathcal{Z}$. Let $\{S(t)\}_{t \geq 0}$ be a family of operators from $H$ into itself satisfying the semigroup properties

$$
\begin{gathered}
S(t+s)=S(t) S(s) \quad \forall s, t \geq 0, \\
S(0)=I
\end{gathered}
$$

and $S(t): \mathbb{R}_{+} \times H \rightarrow H$ is continuous. In the case of a discrete dynamical system then $t \in \mathcal{Z}$ and $S(t) \equiv S^{t}$ where $S: H \rightarrow H$ is a continuous operator so that the semigroup properties are automatic.

For each $v \in H$ we denote the positive orbit

$$
\bigcup_{t \geq 0} S(t) v
$$

by $\gamma^{+}(v)$ and by $\omega(v)$ the $\omega$-limit set defined by

$$
\omega(v)=\bigcap_{s \geq 0} \overline{\bigcup_{t \geq s} S(t) v}
$$

which is equivalent to definition (1.7) with $L^{2}(\Omega)$ replaced by the general metric space $H$. For a set $B \in H$ we define the $\omega$-limit set $\omega(B)$ by

$$
\omega(B)=\bigcap_{s \geq 0} \overline{\bigcup_{t \geq s} S(t) B}
$$

which is equivalent to

$$
\left\{w \in H: \exists t_{j} \rightarrow \infty \text { and } v_{j} \in B: w=\lim _{j \rightarrow \infty} S\left(t_{j}\right) v_{j}\right\}
$$

A set $B$ is said to be invariant if $S(t) B=B$, for all $t \geq 0$. A set $\mathcal{A}$ is said to attract a set $B$ under $S$ if, for any $\varepsilon>0$ there exists $t_{0}=t_{0}(\varepsilon, B, A)$ such that, for $t \geq t_{0}$ $S(t) B \subset N^{\varepsilon}(\mathcal{A})$, an $\varepsilon$-neighbourhood of $A$. A set $B \subset U$, where $U$ is an open set, is said to be absorbing in $U$ under $S$ if for any bounded set $B_{0} \subset U$ there exists $t_{0}=t_{0}\left(B_{0}\right)$ such that $S(t) B_{0} \subset B$ for $t \geq t_{0}$. A set $\mathcal{A}$ is said to be a global attractor for an open set $U \subset H$ under $S$ if
(a) $\mathcal{A}$ is a compact invariant set;
(b) every compact invariant set of $S$ belongs to $\mathcal{A}$;
(c) $\mathcal{A}$ attracts every bounded set $B \subset U$ under $S$. 
We say that $v$ is an equilibrium point of $S$ if $S(t) v=v$ for all $t \geq 0$. The set of all equilibria is denoted by $\mathcal{E}$. A continuous functional $I: H \rightarrow \mathbb{R}$ is said to be a Lyapunov functional on $U$ under $S$ if the following conditions apply.

I(i) There exists $c_{l}$ such that $I(v) \geq c_{l}$ for all $v \in U$, and for every $c$ there exists $\rho=\rho(c)$ such that the diameter of $\{v: I(v) \leq c\}$ is bounded by $\rho$.

I(ii) $I(S(t) v) \leq I\left(S\left(t^{\prime}\right) v\right)$ for all $t \geq t^{\prime}>0$ and for all $v \in U$.

I(iii) If $I(S(t) v)=I(v)$ for all $t>0$ then $v \in \mathcal{E}$.

$S$ is said to be point dissipative on an open set $U \subset H$ if there exists a bounded set $B \subset U$ which attracts each point of $U . S$ is said to be uniformly compact on an open set $U \subset H$ if for each bounded set $B \subset U$ there exists $t_{0}=t_{0}(B)$ such that

$$
\bigcup_{t \geq t_{0}} S(t) B
$$

is relatively compact.

LEMMA 2.1. Let $H$ be finite dimensional and $U \subset H$ be open. Then for either of the properties

(i) there exists a bounded set $B$ which is absorbing in $U$ under $S$, or

(ii) there exists a Lyapunov functional, $I$, on $U$ under $S$ imply that $S$ is uniformly compact on $U$.

Proof. Since $H$ is finite dimensional it is sufficient to show that there exists $t_{0}=t_{0}(B)$ such that, for each bounded set $B_{0} \subset U, \bigcup_{t>t_{0}} S(t) B_{0}$ is bounded. If (i) holds then this is immediate since $\bigcup_{t \geq t_{0}} S(t) B_{0} \subset B$ is a bounded set.

If (ii) holds, by I(iii), we have that for each $v \in B_{0}, I(S(t) v) \leq I(v) \leq M\left(B_{0}\right)$ for all $t>0$ and by $I(i)$ it holds that $S(t) B_{0}$ is bounded for all $t>0$.

LEMMA 2.2. [26, p. 28]. Let $B \subset H$ be nonempty and $\bigcup_{t \geq t_{0}} S(t) B$ be relatively compact for some $t_{0}$. Then $\omega(B)$ is nonempty, compact, and invariant.

Proposition 2.3. [26, p. 23]. Let there exist an open set $U \subset H$ and a bounded set $B \subset U$ such that $B$ is absorbing in $U$ under $S$. Suppose that $S$ is uniformly compact on $U$. Then $\mathcal{A}=\omega(B)$ is a global attractor for $U$ under $S$. Furthermore, if $H$ is a Banach space and $U$ is convex and connected, then $\mathcal{A}$ is connected.

Proposition 2.4. Suppose that $\gamma^{+}\left(u_{0}\right)$ is relatively compact for each $u_{0} \in U$ and that there exists a Lyapunov functional I on $U$ under $S$. Then $\omega\left(u_{0}\right) \subset \mathcal{E}$ for each $u_{0} \in U$. Furthermore, if $\mathcal{E}$ is bounded then $S$ is point dissipative on $U$.

Proof. By I(i) and I(iii), $I\left(S(t) u_{0}\right)$ is a bounded decreasing continuous functional for $t \in(0, \infty)$ (or sequence for $t \in \mathcal{Z}$ ) and so has a unique limit

$$
e=\lim _{t \rightarrow \infty} I\left(S(t) u_{0}\right)
$$

Because $\gamma^{+}\left(u_{0}\right)$ is relatively compact there exists a cluster point $v \in \omega\left(u_{0}\right)=$ $\lim _{k \rightarrow \infty} S\left(t_{k}\right) u_{0}$ and, because $I$ is continuous, $I(v)=\lim _{k \rightarrow \infty} I\left(S\left(t_{k}\right) u_{0}\right)=e$. Thus $I(v)=e$ for all $v \in \omega\left(u_{0}\right)$. The invariance of $\omega\left(u_{0}\right)$ (Lemma 2.2) implies that for $v \in \omega\left(u_{0}\right) S(t) v \in \omega\left(u_{0}\right)$ for all $t \geq 0$. Therefore, $I(S(t) v)=e=I(v)$ for all $t \geq 0$ and, by I(iii) it follows that $v \in \mathcal{E}$.

We have for each $u_{0} \in U$ that

$$
\lim _{t \rightarrow \infty} I\left(S(t) u_{0}\right)=e,
$$

which implies that for $t \geq t_{0}=t_{0}\left(u_{0}\right), I\left(S(t) u_{0}\right) \leq e+1$. Therefore, by I(i) there exists a bounded set $B$ such that $S(t) u_{0} \in B$ for all $t \geq t_{0}\left(u_{0}\right)$. Thus $S$ is point dissipative.

Proposition 2.5. Suppose that 
(i) $S$ has a Lyapunov functional I on $U$.

(ii) $S$ is uniformly compact on $U$.

(iii) $\mathcal{E}$ is bounded.

Then there exists a global attractor $\mathcal{A}$ for $U$ under $S$. Furthermore, if $H$ is a Banach space and $U$ is convex and connected, then $\mathcal{A}$ is connected.

Proof. It follows from Proposition 2.4 that $S$ is point dissipative on $U$. Furthermore, by I(ii) and I(iii) the orbits of bounded sets are bounded. The existence of a connected global attractor is then a consequence of [8, p. 39, Thm. 3.4.6].

3. The semidiscrete approximation: spurious steady solutions. In this section we study the ordinary differential equations (1.8)-(1.9). We start by introducing some notation and making explicit some assumptions about the structure of the spatial discretization. We introduce a discrete $L^{2}$ inner product

$$
\langle\mathbf{U}, \mathbf{V}\rangle=\mathbf{U}^{T} M \mathbf{V}
$$

and a discrete Dirichlet inner product

$$
\langle\mathbf{U}, \mathbf{V}\rangle_{A}=\mathbf{U}^{T} A \mathbf{V}
$$

together with the associated norms

$$
|\mathbf{U}|=\langle\mathbf{U}, \mathbf{U}\rangle^{\frac{1}{2}},\|\mathbf{U}\|=\langle\mathbf{U}, \mathbf{U}\rangle_{A}^{\frac{1}{2}} .
$$

It is convenient to let $L_{2}^{h}$ be the normed vector space $\left\{\mathbb{R}^{J},|\bullet|\right\}$. We assume that the eigenvalue problem

$$
A \psi_{i}=\lambda_{i} M \psi_{i}
$$

has eigenvalues $\lambda_{i}$ satisfying

$$
C_{p}^{-2} \leq \lambda_{i} \leq C_{0} h^{-2}
$$

Under this assumption it is possible to show (see Appendix 1) that the following Poincaré and inverse inequalities hold for all $\mathbf{U} \in \mathbb{R}^{J}$, where $C_{p}$ and $C_{0}$ are independent of $h$,

$$
\begin{gathered}
|\mathbf{U}| \leq C_{p}\|\mathbf{U}\|, \\
\|\mathbf{U}\|^{2} \leq \frac{C_{0}}{h^{2}}|\mathbf{U}|^{2}, \\
\|\mathbf{U}\| \leq C_{p}\left|M^{-1} A \mathbf{U}\right|
\end{gathered}
$$

and

$$
\left|M^{-1} A \mathbf{U}\right|^{2} \leq \frac{C_{0}}{h^{2}}\|\mathbf{U}\|^{2}
$$

We set

$$
L=M^{-1} A, \mathbf{E}=(1,1, \ldots, 1)^{T}
$$


and we assume that

$$
0<L_{i i} \leq \frac{C_{1}}{h^{2}}, \quad L_{i j} \leq 0, \quad j \neq i
$$

where $C_{1}$ is independent of $h$. In addition, we assume that

$$
\{A \mathbf{E}\}_{i} \geq 0 \text { for } i=1, \ldots, J .
$$

The assumptions (3.5) and (3.6) imply that

$$
\lambda_{\phi}\langle\mathbf{U}, \phi(\mathbf{U})\rangle_{A} \geq\|\phi(\mathbf{U})\|^{2}
$$

[20] for monotone $\phi$ with Lipschitz constant $\lambda_{\phi}$ satisfying $\phi(0)=0$.

The assumptions that we make about the matrices $M$ and $A$ are reasonable and will be satisfied by standard second order finite difference approximations and piecewise linear finite element discretisations on "acute triangulations" of $\Omega$.

3.1. Equilibrium problem and steady solutions. We say that $\mathbf{U}^{*} \in L^{2}(\Omega)$ is an equilibrium of (1.8) provided

$$
\gamma A \mathbf{U}^{*}+M \mathbf{f}\left(\mathbf{U}^{*}\right)=0
$$

and we let $\mathcal{E}^{h}$ denote the set of all solutions to (3.8). Clearly $0 \in \mathcal{E}^{h}$ so that $\mathcal{E}^{h}$ is nonempty. Furthermore, if $\mathbf{U}^{*} \in \mathcal{E}^{h}$ then $\mathbf{U}^{*}$ is a critical point of the functional

$$
I^{h}(\mathbf{V}):=\frac{\gamma}{2}\langle\mathbf{V}, \mathbf{V}\rangle_{A}+\langle\mathbf{F}(\mathbf{V}), \mathbf{E}\rangle
$$

By (F2) there exists, for each $\varepsilon>0$ a $C_{\varepsilon} \geq 0$ such that

$$
F(r) \geq-\varepsilon r^{2}-C_{\varepsilon} \quad \forall r
$$

(see [8]) and hence it follows that

$$
I^{h}(V) \geq \frac{\gamma}{2}\langle\mathbf{V}, \mathbf{V}\rangle_{A}-\varepsilon|\mathbf{V}|^{2}-C_{\varepsilon} C(\Omega) \geq \frac{\gamma}{4}\|\mathbf{V}\|^{2}-C_{\gamma} \quad \forall \mathbf{V} \in L_{2}^{h},
$$

where $C(\Omega)$ is an $h$-independent upper bound for $|\mathbf{E}|^{2}$. Therefore, since (F1) implies that $I^{h}(\bullet)$ is continuous, classical arguments show that there exists at least one $\mathbf{U}^{*} \in$ $\mathcal{E}^{h}$ such that

$$
I^{h}\left(\mathbf{U}^{*}\right)=\inf _{\mathbf{V} \in L_{2}^{h}} I^{h}(\mathbf{V})
$$

LEMMA 3.1. The set $\mathcal{E}^{h}$ is uniformly bounded, independent of $h$, in the discrete $H_{0}^{1}$ and in the maximum norm.

Proof. Taking the inner product of (3.8) and $\mathbf{U}^{*}$ gives

$$
\gamma\left\|\mathbf{U}^{*}\right\|^{2}+\left\langle\mathbf{U}^{*}, \mathbf{f}\left(\mathbf{U}^{*}\right)\right\rangle=0
$$

and, since by $(\mathrm{F} 1),(\mathrm{F} 2)$

$$
f(r) r \geq \min _{|r| \leq \bar{u}} f(r) r=-C_{f}
$$


it follows that $\mathcal{E}^{h}$ is bounded in the discrete $H_{0}^{1}$ norm, viz

$$
\left.\left\|\mathbf{U}^{*}\right\| \leq \sqrt{(} C_{f} C(\Omega) / \gamma\right) \quad \forall \mathbf{U}^{*} \in \mathcal{E}^{h} .
$$

Furthermore, taking the inner product of $(3.8)$ with $\left(\mathbf{U}^{*}-\bar{u} \mathbf{E}\right)_{+}$, where the + denotes positive part, and using (3.7), it follows that

$$
\left\|\left(\mathbf{U}^{*}-\bar{u} \mathbf{E}\right)_{+}\right\|=0
$$

since

$$
f(r)(r-\bar{u})_{+} \geq 0 \quad \forall r .
$$

Similarly, using - to denote the negative part, we have that

$$
\left\|\left(\mathbf{U}^{*}+\bar{u} \mathbf{E}\right)-\right\|=0
$$

and hence we have that $\mathcal{E}^{h}$ is uniformly bounded in the maximum norm

$$
\left|\mathbf{U}^{*}\right|_{\infty} \leq \bar{u} \quad \forall \mathbf{U}^{*} \in \mathcal{E}^{h}
$$

Our next result concerns the number of steady solutions (equilibria) of (1.8)-(1.9). We make an additional assumption on $f(u)$, namely (F4).

(F4) $f(-1)=f(0)=f(1)=0$ and $f^{\prime}(-1), f^{\prime}(1)>0$ and $f^{\prime}(0)=-1$.

The canonical example of a function with such properties is $f(u)=u^{3}-u$. With this choice of $f$ equation (1.1) is sometimes known as the Ginzburg-Landau or AllenCahn equation. Similar results can be proved if the number of zeros of $f$ is different, with the obvious modification. In the case $f(u)=u^{2}-u[15]$ has numerical examples which illustrate an analogous result. Simultaneously, a result similar to Theorem 3.2 has been proved by [1]. Their proof of existence is identical, but their proof of stability is different, relying on the structure of the discrete Laplacian, leading to explicit estimates for $\gamma_{0}(h)$. A similar method was also used by [23] to study the existence of spurious solutions of the viscous Burgers's equation when the dissipation is small.

In the following, a steady state $\mathbf{U}^{*}$ is said to be asymptotically stable if it is stable in the sense of Lyapunov and, in addition, $\mathbf{U}(t) \rightarrow \mathbf{U}^{*}$ as $t \rightarrow \infty$ for all $\mathbf{U}(0)$ in a neighbourhood of $\mathbf{U}^{*}$.

THEOREM 3.2. Under conditions (F1) and (F4) there exists $\gamma_{0}(h)>0$ such that, for $\gamma \in\left[0, \gamma_{0}(h)\right)(1.8)$ has at least $3^{J}$ steady solutions and $2^{J}$ of these are asymptotically stable.

Proof. Let $\mathbf{W}$ be one of the $3^{J}$ solutions of (3.8) with $\gamma=0$ which are generated by choosing

$$
W_{j}=+1, \quad-1, \quad \text { or } \quad 0 .
$$

To show that each $\mathbf{W}$ generates an equilibrium solution for $\gamma$ sufficiently small, we employ the implicit function theorem. Set

$$
G(\gamma, \mathbf{U}):=\gamma A \mathbf{U}+M \mathbf{f}(\mathbf{U})
$$

Since $f^{\prime}\left(W_{j}\right) \neq 0$ and since $M$ has positive elements we deduce that the Jacobian $D_{U} G$ evaluated at $(0, \mathbf{W})$ is a diagonal matrix with nonzero diagonal entries and 
so is nonsingular. Hence, by the implicit function theorem, we generate a family of solutions $\mathbf{U}^{*}(\gamma)$ parameterised by $\gamma$ for $\gamma \in\left[0, \gamma_{0}\right)$ with $\mathbf{U}^{*}(0)=\mathbf{W}$.

The stability of these steady solutions is governed by the eigenvalues of $D_{U} G(\gamma, \mathbf{U})$. Now consider the $2^{J}$ steady solutions of $(1.8)$ generated by the choice $U_{j}^{*}(0)= \pm 1$. It follows from (F4) that $D_{U} G(0, \mathrm{~W})$ is a diagonal matrix with strictly positive entries defining its eigenvalues. By the implicit function theorem $\mathbf{U}(\gamma)$ is $C^{1}$, and so the eigenvalues of $D_{U} G(\gamma, \mathbf{U}(\gamma))$ depend continuously on $\gamma$ and thus are positive for $\gamma$ sufficiently small. This demonstrates that $2^{J}$ solutions are stable.

Many of these steady states constructed for $\gamma \ll 1$ are spurious and the stability properties of approximations of genuine steady solutions are often incorrect; for example the Ginzburg-Landau equation has only two stable steady states (for $\gamma$ small) whereas the numerical approximation has $2^{J}$ for $\gamma$ sufficiently small. This issue is discussed in detail in $\S 7$, where numerical results are presented.

However, as $h \rightarrow 0$ for fixed $\gamma$ the structure of the discrete steady solution set converges to that of the continuous steady solution set; we refer to [4] for an account of the numerical solution of bifurcation problems. In Appendix 3 we show that when (1.1)-(1.3) has $N$ isolated solutions then the same is also true of (3.8) for $h$ sufficiently small. See also [17].

3.2. Existence of absorbing sets, attractors, and stabilization. The appropriate analogues of (R1)-(R4), which we establish in Theorem 3.4 are stated below; note that $B_{2}^{h}$ is an absorbing set in the discrete $H_{0}^{1}$ norm even though the initial data is only bounded uniformly in $h$ in $\left\{L_{2}^{h},|\bullet|\right\}$.

$(\mathrm{R} 1)^{h}$ For each $\mathbf{U}_{0} \in L_{2}^{h}$ there exists a unique solution of (1.8)-(1.9) $\mathbf{U} \in$ $C^{2}\left([0, T] ; L_{2}^{h}\right)$ for all $T>0$. The mapping $\mathbf{U}_{0} \rightarrow \mathbf{U}(t)$ is continuous in $L_{2}^{h}$ for each $t>0$. Hence the family of solution operators $\left\{S^{h}(t)\right\}_{t \geq 0}$, defined by $S^{h}(t) \mathbf{U}_{0} \equiv \mathbf{U}(t)$, forms a continuous semigroup on $L_{2}^{h}$.

$(\mathrm{R} 2)^{h}$ There exist constants $\left\{\bar{\rho}_{i}\right\}_{i=1}^{2}$ independent of $h$ and $J$ such that the balls

$$
\begin{aligned}
& B_{1}^{h}=\left\{\mathbf{V} \in L_{2}^{h}:|\mathbf{V}| \leq \overline{\rho_{1}}\right\} \\
& B_{2}^{h}=\left\{\mathbf{V} \in L_{2}^{h}:\|\mathbf{V}\| \leq \overline{\rho_{2}}\right\}
\end{aligned}
$$

are absorbing sets for the semigroup $\left\{S^{h}(t)\right\}_{t \geq 0}$; that is, for each $\mathbf{U}_{0} \in L_{2}^{h}$ there exists $\left\{t_{i}\right\}_{i=1}^{2}$ (depending on $\left\{\mathbf{U}_{0}, \rho_{1}\right\}$ and $\left\{\mathbf{U}_{0}, \rho_{2}\right\}$, respectively) such that

$$
S^{h}(t) \mathbf{U}_{0} \in B_{i}^{h} \quad \forall t \geq t_{i} \quad(i=1,2) .
$$

$(\mathrm{R} 3)^{h}$ There exists a global attractor $\mathcal{A}^{h} \subset L_{2}^{h}$ for the semigroup $\left\{S^{h}(t)\right\}_{t \geq 0}$. Furthermore $\mathcal{A}^{h}$ is connected and there exists a constant $\overline{\rho_{A}}$ independent of $h$ such that

$$
\max \left\{|\mathbf{V}|,\|\mathbf{V}\|,|\mathbf{V}|_{\infty}\right\} \leq \overline{\rho_{A}} \quad \forall \mathbf{V} \in \mathcal{A}^{h}
$$

$(\mathrm{R} 4)^{h}$ The functional defined by (3.9) is a Lyapunov functional for $\left\{S^{h}(t)\right\}_{t>0}$. In addition, for any $\mathbf{U}_{0} \in L_{2}^{h}$, the $\omega$-limit set is contained in $\mathcal{E}^{h}$.

We now prove these results.

LEMMA 3.3. Let $m \geq \bar{u}$. If $\mathrm{U}(t)$ solves (1.8)-(1.9) then, for $t>t_{0} \geq 0$

$$
\left|(\mathbf{U}(t)-m \mathbf{E})_{+}\right| \leq\left|\left(\mathbf{U}\left(t_{0}\right)-m \mathbf{E}\right)_{+}\right| \exp \left(-\left(t-t_{0}\right) \gamma / C_{p}^{2}\right),
$$




$$
\left|(\mathbf{U}(t)+m \mathbf{E})_{-}\right| \leq\left|\left(\mathbf{U}\left(t_{0}\right)+m \mathbf{E}\right)_{-}\right| \exp \left(-\left(t-t_{0}\right) \gamma / C_{p}^{2}\right),
$$

and

$$
|\mathbf{U}(t)|_{\infty} \leq \max \left(\bar{u},\left|\mathbf{U}_{0}\right|_{\infty}\right)
$$

Proof. Set

$$
\mathbf{Z}(t)=\mathbf{U}(t)-m \mathbf{E}
$$

and define $\mathbf{Z}(t)_{+}$by $\left\{\mathbf{Z}(t)_{+}\right\}_{i}=Z_{i}(t)_{+}$. It follows that

$$
\left\langle\frac{d \mathbf{Z}}{d t}, \mathbf{Z}_{+}\right\rangle+\gamma\left\langle\mathbf{Z}, \mathbf{Z}_{+}\right\rangle_{A}+\left\langle\mathbf{f}(\mathbf{U}), \mathbf{Z}_{+}\right\rangle=-m\left\langle\mathbf{E}, \mathbf{Z}_{+}\right\rangle_{A} .
$$

Since $m \geq \bar{u}$ we have

$$
f(r)(r-m)_{+} \geq 0 \quad \forall r .
$$

Combining the assumptions (3.6), (3.7) on the matrix $A$ yields

$$
\frac{1}{2} \frac{d}{d t}\left|\mathbf{Z}_{+}\right|^{2}+\gamma\left\|\mathbf{Z}_{+}\right\|^{2} \leq 0
$$

Inequality (3.13) is an immediate consequence of (3.16) and the Gronwall inequality. The proof of (3.14) is very similar. We obtain the maximum norm bound (3.15) by taking $m=\max \left(\bar{u},\left|\mathbf{U}_{0}\right|_{\infty}\right)$ and $t_{0}=0$ in (3.13) and (3.14).

THEOREM 3.4. $(\mathrm{R} 1)^{h}-(\mathrm{R} 4)^{h}$ hold for (1.8)-(1.9).

Proof. $(\mathrm{R} 1)^{h}$ : local existence and uniqueness follows from the standard theory of ordinary differential equations, by (F1). Existence for any $T>0$ follows from the a priori estimate (3.15). In order to prove continuity with respect to initial data, it is sufficient to consider $\mathbf{U}(t)-\mathbf{V}(t)$ where $\mathbf{V}(t)$ solves (1.8) with initial data $\mathbf{V}_{0}$. Clearly

$$
\frac{1}{2} \frac{d}{d t}|\mathbf{U}-\mathbf{V}|^{2}+\gamma\|\mathbf{U}-\mathbf{V}\|^{2}+\langle\mathbf{f}(\mathbf{U})-\mathbf{f}(\mathbf{V}), \mathbf{U}-\mathbf{V}\rangle=0
$$

and the inequality

$$
|\mathbf{U}(t)-\mathbf{V}(t)| \leq C\left(T, \mathbf{U}_{0}, \mathbf{V}_{0}\right)\left|\mathbf{U}_{0}-\mathbf{V}_{0}\right|
$$

follows from the a priori estimate (3.15) in Lemma 3.2 and Gronwall's inequality.

$(\mathrm{R} 2)^{h}$ : taking the inner product of $(3.8)$ with $\mathbf{U}(t)$ implies that

$$
\frac{1}{2} \frac{d}{d t}|\mathbf{U}(t)|^{2}+\gamma\|\mathbf{U}\|^{2}+\langle\mathbf{U}, \mathbf{f}(\mathbf{U})\rangle=0
$$

and, by (3.12),

$$
\frac{d}{d t}|\mathbf{U}|^{2}+2 \gamma\|\mathbf{U}\|^{2} \leq 2 C_{f} C(\Omega) .
$$

Using Poincaré's inequality (3.1),

$$
\frac{d}{d t}|\mathbf{U}|^{2}+\frac{2 \gamma}{C_{p}^{2}}|\mathbf{U}|^{2} \leq 2 C_{f} C(\Omega)
$$


From this we obtain, using Gronwall's inequality,

$$
|\mathbf{U}(t)|^{2} \leq\left|\mathbf{U}_{0}\right|^{2} \exp \left(-2 \gamma t / C_{p}^{2}\right)+C_{f} C(\Omega) C_{p}^{2}\left[1-\exp \left(-2 \gamma t / C_{p}^{2}\right)\right] / \gamma .
$$

Therefore,

$$
\limsup _{t \rightarrow \infty}|\mathbf{U}(t)| \leq \delta_{1}, \quad \delta_{1}^{2}=C_{f} C(\Omega) C_{p}^{2} / \gamma
$$

and we can take $\overline{\rho_{1}}$ to be any number larger than $\delta_{1}$ to define the absorbing set $B_{1}^{h}$.

In order to show that there is an absorbing set uniformly bounded in the discrete $H_{0}^{1}$ norm we premultiply equation (1.8) by $M^{-1}$ and take the $\langle\bullet, \bullet\rangle_{A}$ inner product with $\mathbf{U}$ to obtain

$$
\frac{1}{2} \frac{d}{d t}\|\mathbf{U}\|^{2}+\gamma\left|M^{-1} A \mathbf{U}\right|^{2}+\langle\mathbf{f}(\mathbf{U}), \mathbf{U}\rangle_{A}=0
$$

so that

$$
\frac{1}{2} \frac{d}{d t}\|\mathbf{U}\|^{2}+\left\langle\mathbf{f}(\mathbf{U})+C_{F} \mathbf{U}, \mathbf{U}\right\rangle_{A}-\left\langle C_{F} \mathbf{U}, \mathbf{U}\right\rangle_{A}=0 .
$$

Using the monotonicity of $\mathbf{f}(\mathbf{U})+C_{F} \mathbf{U}$ implied by (F3) and (3.7) we obtain

$$
\frac{1}{2} \frac{d}{d t}\|\mathbf{U}\|^{2} \leq C_{F}\|\mathbf{U}\|^{2}
$$

Integrating (3.17) we obtain

$$
\int_{t}^{t+r}\|\mathbf{U}(s)\|^{2} d s \leq \frac{C_{f} C(\Omega) r}{\gamma}+\frac{1}{2 \gamma}|\mathbf{U}(t)|^{2} .
$$

Taking $t$ sufficiently large and using the absorbing set in $L_{2}^{h}$ gives

$$
\int_{t}^{t+r}\|\mathbf{U}(s)\|^{2} d s \leq \frac{C_{f} C(\Omega) r}{\gamma}+\frac{{\overline{\rho_{1}}}^{2}}{2 \gamma} .
$$

Thus, applying the uniform Gronwall lemma [26, p. 89] we obtain

$$
\|\mathbf{U}(t+r)\|^{2} \leq\left[\frac{C_{f} C(\Omega)}{\gamma}+\frac{\overline{\rho_{1}}}{2 \gamma r}\right] \exp \left(2 C_{F} r\right) .
$$

Since $t$ may be arbitrarily large, this yields the absorbing set in the $\|\bullet\|$ norm.

$(\mathrm{R} 3)^{h}$ : We set ourselves in the framework of $\S 2$. Since $L_{2}^{h}$ is finite dimensional, the existence of a global attractor $\mathcal{A}^{h}=\omega^{h}\left(B_{1}^{h}\right)$ is an immediate consequence of Proposition 2.3. The existence of $\bar{\rho}_{A}$ is an immediate consequence of the bounds obtained in the proof of $(\mathrm{R} 2)^{h}$ and from the following maximum norm bound: since

$$
\left|\left(\mathbf{U}\left(t_{0}\right)-m \mathbf{E}\right)_{+}\right| \leq\left|\mathbf{U}\left(t_{0}\right)\right|+m C(\Omega),
$$

it follows from (3.13),(3.14) in Lemma 3.3 with $m=\bar{u}$ and the previous estimate on the absorbing set in the $L_{2}^{h}$ norm that

$$
\limsup _{t \rightarrow \infty}|\mathbf{U}(t)|_{\infty} \leq \bar{u}
$$

$(\mathrm{R} 4)^{h}$ : Taking the inner product of (1.8) with $d \mathbf{U} / d t$ yields

$$
\left|\frac{d \mathbf{U}}{d t}\right|^{2}+\frac{d}{d t} I^{h}(\mathbf{U})=0 .
$$

Facts $I(i)-I(i i i)$ concerning $I^{h}(\bullet)$ follow immediately from (3.11) and (3.18). Thus Proposition 2.3 yields $\omega\left(\mathbf{U}_{0}\right) \subset \mathcal{E}^{h}$. 
4. The explicit Euler approximation. In this section we consider the Euler discretisation of (1.8)-(1.9). Let $\mathbf{U}_{n}$ denote the approximation to $\mathbf{U}(n \Delta t)$. We obtain, for $n \geq 1$,

$$
M \partial \mathbf{U}_{n+1}+\gamma A \mathbf{U}_{n}+M \mathbf{f}\left(\mathbf{U}_{n}\right)=0
$$

where

$$
\partial \mathbf{V}_{n}:=\left(\mathbf{V}_{n}-\mathbf{V}_{n-1}\right) / \Delta t
$$

The convergence of solutions of (4.1) to solutions of (1.1) on a finite time interval as $h, \Delta t \rightarrow 0$ is well understood; see [21]. Such results require that $\Delta t$ be restricted by the square of the space step $h$. Here we are concerned with the long time dynamics of (4.1), that is $n \rightarrow \infty$, with fixed $\Delta t$ and $h$. We show that the temporal discretisation (4.1) introduces spurious solutions with period 2 in the timestep and that, furthermore, under certain assumptions on $f(u)$, these periodic solutions can be found for $\Delta t$ arbitrarily small. We then discuss the effect of these periodic solutions on the dynamics of the problem. If $\Delta t$ is restricted in terms of the initial data then the effect of the periodic solutions is avoided, a gradient structure ensues and the existence of absorbing sets and attractors is proved. However, if $\Delta t$ is not restricted sufficiently in terms of the initial data the scheme may be shown to blow up, indicating that the unstable manifolds of the spurious periodic solutions are connected with infinity.

4.1. Spurious dynamics. Period 2 solutions of (4.1) are pairs $\{\mathbf{U}, \mathbf{V}\}$ with $\mathbf{U} \neq \mathbf{V}$ which satisfy the equations

$$
\begin{aligned}
& M(\mathbf{V}-\mathbf{U})+\Delta t \gamma A \mathbf{U}+\Delta t M \mathbf{f}(\mathbf{U})=0 \\
& M(\mathbf{U}-\mathbf{V})+\Delta t \gamma A \mathbf{V}+\Delta t M \mathbf{f}(\mathbf{V})=0
\end{aligned}
$$

Existence of period 2 solutions can be established by a local bifurcation argument, treating $\Delta t$ as a bifurcation parameter. See [7] and [24]. Fixed points (i.e., equilibria of (4.1)) are solutions of (4.2)-(4.3) with the symmetry $\mathbf{U}=\mathbf{V}$. Genuine period 2 solutions can by constructed as local bifurcations which break this symmetry as $\Delta t$ varies.

Suppose that $\mathbf{U}^{*}$ is an equilibrium solution and let $d \mathbf{f}(\bullet)$ denote the Jacobian of f so that

$$
d \mathbf{f}\left(\mathbf{U}^{*}\right)=\operatorname{diag}\left(f^{\prime}\left(U_{i}^{*}\right)\right) .
$$

Applying Theorem 4.1 in [24] we obtain the following result.

LEMMA 4.1. Let $\mathbf{U}^{*}$ be an equilibrium point of (4.1) (i.e., a solution of (3.8)) such that all the eigenvalues of $\gamma L+d \mathbf{f}\left(\mathbf{U}^{*}\right)$ are nonzero. Assume that $(\mathrm{F} 1)$ holds and $d=1$. Then the eigenvectors $\left\{\phi_{k}\right\}_{k=1}^{J}$ and distinct eigenvalues $\left\{\eta_{k}\right\}_{k=1}^{J}$ of $\gamma L+d \mathbf{f}\left(\mathbf{U}^{*}\right)$ generate period 2 solutions of (4.1) with the form

$$
\begin{gathered}
\mathbf{U}(\mu):=\mathbf{U}^{*}+\mu \phi_{k}+\mathcal{O}\left(|\mu|^{2}\right) \\
\mathbf{V}(\mu):=\mathbf{U}^{*}-\mu \phi_{k}+\mathcal{O}\left(|\mu|^{2}\right) \\
\Delta t_{k}(\mu):=2 / \eta_{k}+\mathcal{O}(|\mu|)
\end{gathered}
$$


for $\mu$ sufficiently small, which are $C^{1}$ in $\mu$.

Note that most of the $\eta_{k}$ are positive since the largest eigenvalues of $L=M^{-1} A$ scale like $h^{-2}$. Here $\mu$ parameterises the branches of period 2 solutions locally in the neighbourhood of the bifurcation points. Having constructed these branches of spurious solutions locally in the neighbourhood of the bifurcation points $\Delta t_{k}$, it is natural to ask what happens to these branches of solutions far from the bifurcation points. In particular, it is of interest to know whether such solutions exist for $\Delta t$ arbitrarily small. For simplicity we consider the case of $f(\bullet)$ being odd. It is then possible to seek period 2 solutions with the symmetry $U=-V$ under which (4.2),(4.3) become

$$
2 \mathbf{U}-\Delta t \mathbf{f}(\mathbf{U})-\Delta t \gamma L \mathbf{U}=0 .
$$

Setting $\gamma=0$, equation (4.4) admits solutions $\mathbf{W}$ with the form

$$
\{\mathbf{W}\}_{i}= \pm R(\Delta t)
$$

where $R(\Delta t)$ solves

$$
r=\Delta t f(r) / 2 \text {. }
$$

Suppose that $f$ satisfies (F1), (F2) and that, in addition,

$$
|f(r) / r| \rightarrow \infty \text { as } r \rightarrow \infty,
$$

then (4.6) has solutions $\pm R(\Delta t)$ for arbitrarily small $\Delta t$ which satisfy

$$
\lim _{\Delta t \rightarrow 0}|R(\Delta t)|=\infty .
$$

These two solutions generate $2^{J}$ solutions of (4.4), and hence $2^{J}$ period 2 solutions of (4.1) in the case of zero diffusion $(\gamma=0)$. These solutions exist for $\Delta t$ arbitrarily small but, in accordance with the results of [13], they tend to infinity as $\Delta t \rightarrow 0$. We now show that these solutions persist for $\gamma>0$ sufficiently small under appropriate conditions on $f(u)$. These conditions allow, for example, arbitrary superlinear polynomial power growth. In the following theorem the norm $|\bullet|$ on matrices denotes that subordinate to the related norm on vectors.

THEOREM 4.2. Let $f$ satisfy (F1), (F2), (F5) and be odd. Assume that $f$ satisfies the following two growth conditions: there exists $\overline{\Delta t}$ satisfying $0<\overline{\Delta t}<1$ :

(i) $\left|2-\Delta t f^{\prime}(R(\Delta t))\right| \geq d_{1}>0$, for all $0 \leq \Delta t \leq \overline{\Delta t}$, where $d_{1}$ is independent of $\Delta t$ and $R(\Delta t)$ solves equation (4.6);

(ii) define

$$
D=2 \mathbf{I d}-\Delta t d \mathbf{f}(\mathbf{W}),
$$

where Id is the identity matrix and note that $D^{-1}$ is defined for $0 \leq \Delta t \leq \overline{\Delta t}$ by (i). Let

$$
\mathcal{B}:=\{\mathbf{V}:|\mathbf{V}-\mathbf{W}| \leq \rho\}
$$

where

$$
\rho=\gamma\left|L \| D^{-1}\right||\mathbf{W}| .
$$

Then we assume $\Delta t K \leq d_{2}$ for all $\Delta t \leq \overline{\Delta t}$ where $K$ is the Lipschitz constant for $d \mathbf{f}$ on $\mathcal{B}$. 
Assume also that $\gamma$ is chosen sufficiently small so that

$$
d_{2}\left|D^{-1}\right| \rho+\bar{\Delta} t\left(1+\gamma|L|\left|D^{-1}\right|\right) \leq 1
$$

and

$$
2 d_{2}\left|D^{-1}\right| \rho+\bar{\Delta} t \gamma|L|\left|D^{-1}\right|<1 .
$$

Then, for each of the $2^{J}$ solutions $\mathbf{W}$ defined by (4.5),(4.6), there exists a unique solution $\mathbf{U}, \mathbf{V}$ to equations (4.2), (4.3) satisfying $\mathbf{V}=-\mathbf{U}$ and

$$
|\mathbf{U}-\mathbf{W}| \leq \rho .
$$

Proof. Solutions of (4.4) are fixed points of the iteration

$$
\mathbf{U}_{k+1}=\mathbf{U}_{k}-D^{-1}\left[2 \mathbf{U}_{k}-\Delta t \mathbf{f}\left(\mathbf{U}_{k}\right)-\Delta t \gamma L \mathbf{U}_{k}\right] .
$$

By (i), this map is well-defined and $\left|D^{-1}\right|$ is bounded independently of $\Delta t$.

We first show that the map takes $\mathcal{B}$ into itself. Let $\mathbf{U}_{k} \in \mathcal{B}$. Clearly,

$$
\mathbf{W}=\mathbf{W}-D^{-1}[2 \mathbf{W}-\Delta t \mathbf{f}(\mathbf{W})] .
$$

Subtracting and exploiting the diagonal nature of $\mathbf{f}$ we obtain

$$
\mathbf{e}_{k+1}=-\Delta t D^{-1}\left[\left(d \mathbf{f}(\mathbf{W})-d \mathbf{f}\left(\xi_{k}\right)\right) \mathbf{e}_{k}-\gamma L \mathbf{U}_{k}\right],
$$

where $\mathbf{e}_{k}=\mathbf{U}_{k}-\mathbf{W}$ and $\xi_{k} \in \mathcal{B}$. Using the Lipschitz constant for $d \mathbf{f}$ we find that

$$
\begin{aligned}
\left|\mathbf{e}_{k+1}\right| & \leq \Delta t\left|D^{-1}\right| K\left|\mathbf{e}_{k}\right|^{2}+\Delta t \gamma|L|\left|D^{-1}\right||\mathbf{W}|+\Delta t \gamma|L|\left|D^{-1}\right|\left|\mathbf{e}_{k}\right| \\
& \leq d_{2}\left|D^{-1}\right| \rho^{2}+\Delta t \rho\left(1+\gamma|L|\left|D^{-1}\right|\right) \\
& \leq\left[d_{2}\left|D^{-1}\right| \rho+\Delta t\left(1+\gamma\left|L \| D^{-1}\right|\right)\right] \rho
\end{aligned}
$$

and hence, by assumption on $\gamma$ the mapping takes $\mathcal{B}$ into itself.

Now consider a second sequence of iterates $\mathbf{V}_{k}$ satisfying the same iteration scheme and assume that $\mathbf{U}_{0}$ and $\mathbf{V}_{0} \in \mathcal{B}$. Defining $\mathbf{d}_{k}=\mathbf{U}_{k}-\mathbf{V}_{k}$ we obtain

$$
\mathbf{d}_{k+1}=-\Delta t D^{-1}\left[\left(d \mathbf{f}(\mathbf{W})-d \mathbf{f}\left(\eta_{k}\right)\right) \mathbf{d}_{k}-\gamma L \mathbf{d}_{k}\right],
$$

where $\eta_{k} \in \mathcal{B}$. Thus

$$
\begin{aligned}
\left|\mathbf{d}_{k+1}\right| & \leq \Delta t\left|D^{-1}\right| K\left|\mathbf{d}_{k}\right|^{2}+\Delta t \gamma|L|\left|D^{-1}\right|\left|\mathbf{d}_{k}\right| \\
& \leq\left[2 d_{2}\left|D^{-1}\right| \rho+\Delta t \gamma|L|\left|D^{-1}\right|\right]\left|\mathbf{d}_{k}\right| .
\end{aligned}
$$

Hence, by assumption on $\gamma$ the mapping is a contraction on $\mathcal{B}$. By the contraction mapping theorem, the proof is complete.

We now consider the effect of these spurious period 2 solutions on the dynamics of the Euler scheme. It is clear that, if the scheme is to inherit the gradient structure of the underlying PDE, then some restriction will be required on $\Delta t$ in terms of the magnitude of the initial data - this is because for any $\Delta t$ sufficiently small, it is possible to choose initial data in the domain of attraction of a period 2 solution which precludes stabilisation to the set of equilibria and hence a gradient structure cannot hold. Furthermore, we conjecture that the unstable manifolds of the period 2 solutions 
are connected to infinity. To substantiate this conjecture, we prove that for all $\Delta t$ sufficiently small there is initial data which blows up under the Euler iteration (4.1). This should be contrasted with the underlying partial differential equation and its semidiscretisation, for which arbitrarily large initial data is mapped into an absorbing set under the evolution semigroup.

The following relationships are needed in the analysis:

$$
|\mathbf{U}| \leq C(\Omega)|\mathbf{U}|_{\infty} \quad \forall U \in \mathcal{R}^{J}
$$

and

$$
|\mathbf{U}|^{2} \geq C_{2} h^{d}|\mathbf{U}|_{\infty}^{2} \quad \forall U \in \mathcal{R}^{J}
$$

Here $C_{2}$ is independent of $h$, and $d$ is the spatial dimension of the problem. In the following set $r=\Delta t / h^{2}$.

THEOREM 4.3. Consider (4.1) under conditions (F1), (F2), and (F5) with initial data $\mathbf{U}_{0}$ satisfying the property that $|f(u)|>C_{3}|u|$ for all $u:|u| \geq\left|\mathbf{U}_{0}\right|_{\infty}$, where $C_{3}, h$ and $r$ are chosen so that

$$
\begin{aligned}
& \Delta t C_{3}\left[\Delta t C_{2} h^{d} C_{3}-2 C(\Omega)^{2}-2 r C_{0} \gamma C(\Omega)^{2}\right] \\
& \quad \geq C(\Omega)^{2}(1+k)-\left(1-2 r \gamma C_{0}+\frac{\Delta t^{2} \gamma^{2}}{C_{p}^{4}}\right) C_{2} h^{d},
\end{aligned}
$$

for some $k>0$ and

$$
\Delta t C_{3}\left[\Delta t C_{2} h^{d} C_{3}-2 C(\Omega)^{2}-2 r C_{0} \gamma C(\Omega)^{2}\right] \geq 0 .
$$

Then

$$
\left|\mathbf{U}_{n}\right|_{\infty}^{2} \geq(1+k)^{n}\left|\mathbf{U}_{0}\right|_{\infty}^{2}
$$

Proof. The proof is by induction. Rearranging (4.1) gives

$$
M \mathbf{U}_{n+1}=M \mathbf{U}_{n}-\Delta t \gamma A \mathbf{U}_{n}-\Delta t M \mathbf{f}\left(\mathbf{U}_{n}\right) .
$$

Taking the inner product of each side of this equation with itself yields

$$
\begin{aligned}
\left|\mathbf{U}_{n+1}\right|^{2}= & \left|\mathbf{U}_{n}\right|^{2}-2 \Delta t \gamma\left\|\mathbf{U}_{n}\right\|^{2}-2 \Delta t\left\langle\mathbf{U}_{n}, \mathbf{f}\left(\mathbf{U}_{n}\right)\right\rangle \\
& +2 \Delta t^{2} \gamma\left\langle\mathbf{f}\left(\mathbf{U}_{n}\right), \mathbf{U}_{n}\right\rangle_{A}+\Delta t^{2} \gamma^{2}\left|M^{-1} A \mathbf{U}_{n}\right|^{2}+\Delta t^{2}\left|\mathbf{f}\left(\mathbf{U}_{n}\right)\right|^{2} .
\end{aligned}
$$

Using (3.1), (3.2), (3.3), (4.7), and (4.8) we obtain

$$
\begin{aligned}
& \left|\mathbf{U}_{n+1}\right|^{2} \\
& \geq\left(1-2 r \gamma C_{0}+\frac{\Delta t^{2} \gamma^{2}}{C_{p}^{4}}\right)\left|\mathbf{U}_{n}\right|^{2}-2 \Delta t\left|\mathbf{U}_{n}\right|\left|\mathbf{f}\left(\mathbf{U}_{n}\right)\right| \\
& -2 \Delta t^{2} \gamma\left\|\mathbf{U}_{n}\right\|\left\|\mathbf{f}\left(\mathbf{U}_{n}\right)\right\|+\Delta t^{2}\left|\mathbf{f}\left(\mathbf{U}_{n}\right)\right|^{2} \\
& \geq\left(1-2 r \gamma C_{0}+\frac{\Delta t^{2} \gamma^{2}}{C_{p}^{4}}\right)\left|\mathbf{U}_{n}\right|^{2} \\
& -2 \Delta t\left|\mathbf{U}_{n}\right|\left|\mathbf{f}\left(\mathbf{U}_{n}\right)\right|-2 \Delta t r C_{0} \gamma\left|\mathbf{U}_{n}\right|\left|\mathbf{f}\left(\mathbf{U}_{n}\right)\right|+\Delta t^{2}\left|\mathbf{f}\left(\mathbf{U}_{n}\right)\right|^{2}
\end{aligned}
$$




$$
\begin{aligned}
\geq & \left(1-2 r \gamma C_{0}+\frac{\Delta t^{2} \gamma^{2}}{C_{p}^{4}}\right)\left|\mathbf{U}_{n}\right|^{2}-2 \Delta t C(\Omega)^{2}\left|\mathbf{U}_{n}\right|_{\infty}\left|\mathbf{f}\left(\mathbf{U}_{n}\right)\right|_{\infty} \\
& -2 \Delta t r C_{0} \gamma C(\Omega)^{2}\left|\mathbf{U}_{n}\right|_{\infty}\left|\mathbf{f}(\mathbf{U})_{n}\right|_{\infty}+\Delta t^{2} C_{2} h^{d}\left|\mathbf{f}\left(\mathbf{U}_{n}\right)\right|_{\infty}^{2} \\
= & \left(1-2 r \gamma C_{0}+\frac{\Delta t^{2} \gamma^{2}}{C_{p}^{4}}\right)\left|\mathbf{U}_{n}\right|^{2}+\Delta t\left|\mathbf{f}\left(\mathbf{U}_{n}\right)\right|_{\infty}\left[\Delta t C_{2} h^{d}\left|\mathbf{f}\left(\mathbf{U}_{n}\right)\right|_{\infty}\right. \\
& \left.-2 C(\Omega)^{2}\left|\mathbf{U}_{n}\right|_{\infty}-2 r C_{0} \gamma C(\Omega)^{2}\left|\mathbf{U}_{n}\right|_{\infty}\right] .
\end{aligned}
$$

Assume for the purposes of induction that $\left|\mathbf{U}_{n}\right|_{\infty}$ satisfies

$$
\left|\mathbf{U}_{n}\right|_{\infty} \geq(1+k)^{n / 2}\left|\mathbf{U}_{0}\right|_{\infty} .
$$

Now

$$
\left|\mathbf{f}\left(\mathbf{U}_{n}\right)\right|_{\infty} \geq|f(v)|
$$

where $|v|=\left|\mathbf{U}_{n}\right|_{\infty}$ and hence

$$
\left|\mathbf{f}\left(\mathbf{U}_{n}\right)\right|_{\infty}>C_{3}\left|\mathbf{U}_{n}\right|_{\infty}
$$

By assumption on $C_{3}$ it follows that

$$
\Delta t C_{2} h^{d} C_{3}-2 C(\Omega)^{2}-2 r C_{0} \gamma C(\Omega)^{2}>0 .
$$

Thus we have

$$
\begin{aligned}
\left|\mathbf{U}_{n+1}\right|^{2} \geq & \left(1-2 r \gamma C_{0}+\frac{\Delta t^{2} \gamma^{2}}{C_{p}^{4}}\right)\left|\mathbf{U}_{n}\right|^{2} \\
& +\Delta t C_{3}\left[\Delta t C_{2} h^{d} C_{3}-2 C(\Omega)^{2}-2 r C_{0} \gamma C(\Omega)^{2}\right]\left|\mathbf{U}_{n}\right|_{\infty}^{2} \\
\geq & C_{2} h^{d}\left(1-2 r \gamma C_{0}+\frac{\Delta t^{2} \gamma^{2}}{C_{p}^{4}}\right)\left|\mathbf{U}_{n}\right|_{\infty}^{2} \\
& +\Delta t C_{3}\left[\Delta t C_{2} h^{d} C_{3}-2 C(\Omega)^{2}-2 r C_{0} \gamma C(\Omega)^{2}\right]\left|\mathbf{U}_{n}\right|_{\infty}^{2} .
\end{aligned}
$$

By assumption this yields

$$
\left|\mathbf{U}_{n+1}\right|^{2} \geq C(\Omega)^{2}(1+k)\left|\mathbf{U}_{n}\right|_{\infty}^{2} .
$$

Hence,

$$
\left|\mathbf{U}_{n+1}\right|_{\infty}^{2} \geq(1+k)\left|\mathbf{U}_{n}\right|_{\infty}^{2}
$$

Thus, since $k>0,(4.9)$ holds for $n=n+1$ and the induction is complete.

The magnitude of the data required to prove blowup in Theorem 4.3 is very large: a rough estimate shows that $C_{3}=\mathcal{O}\left(\Delta t^{-1} h^{-d / 2}\right)$. The analysis is very crude since it is based in the maximum norm and no attempt is made to determine the most unstable spatial structure required for blowup. In the case of one-dimensional finite difference schemes, the analysis can be tightened to yield a critical $C_{3}=\mathcal{O}\left(\Delta t^{-1}\right)$. In this case a scheme of the form (4.1) may be written

$$
U_{j}^{n+1}=r U_{j-1}^{n}+(1-2 r) U_{j}^{n}+r U_{j+1}^{n}-\Delta t f\left(U_{j}^{n}\right) \text { for } j=1, \ldots, J,
$$

together with the boundary conditions

$$
U_{0}^{n}=U_{J+1}^{n}=0
$$


Consider initial data $U_{j}^{0}$ satisfying

$$
U_{l}^{0} U_{l+1}^{0}<0 \quad \forall l=1, \ldots, J-1 .
$$

This choice of initial data is very unstable and is motivated by the most unstable mode according to linear theory [21]. Note that the condition we derive for blowup may. be reformulated as

$$
\Delta t>\frac{(2+k) h^{2}}{2+h^{2} C}
$$

where $C$ is defined below. This should be contrasted with the bound (4.15) on $\Delta t$ in Lemma 4.5 which ensures boundedness of the numerical solution as $n \rightarrow \infty$.

THEOREM 4.4. Consider (4.10)-(4.11) under conditions (F1), (F2), and (F5) together with initial data satisfying (4.12). In addition, assume that the initial data satisfies the property that $f(u) / u>C$ for all $u:|u| \geq \min _{j}\left|\left\{\mathbf{U}_{0}\right\}_{j}\right|$ and where $C$ is chosen so that

$$
C \geq \frac{2-2 r+k}{\Delta t}
$$

for some $k>0$. Then

$$
\left|\mathbf{U}_{n}\right|_{\infty} \geq(1+k)^{n}\left|\mathbf{U}_{0}\right|_{\infty}
$$

Proof. The proof proceeds by induction. We assume that

$$
U_{l}^{n} U_{l+1}^{n}<0 \quad \forall l=1, \ldots, J-1 .
$$

and that

$$
f\left(U_{j}^{n}\right) / U_{j}^{n}>C \text { for } j=1, \ldots J .
$$

Both (4.13) and (4.14) hold by assumption for $n=0$. Consider any $U_{j}^{n}>0$. From (4.10) and (4.13) we deduce that

$$
U_{j}^{n+1} \leq(1-2 r) U_{j}^{n}-\Delta t f\left(U_{j}^{n}\right) .
$$

Hence, by (4.14),

$$
\begin{aligned}
U_{j}^{n+1} & \leq\left[1-2 r-\Delta t \frac{f\left(U_{j}^{n}\right)}{U_{j}^{n}}\right] U_{j}^{n} \\
& \leq[1-2 r-\Delta t C] U_{j}^{n} .
\end{aligned}
$$

By assumption on $C$ we obtain

$$
U_{j}^{n+1} \leq-(1+k) U_{j}^{n}
$$

Similarly, for $U_{j}^{n}<0$, we obtain

$$
U_{j}^{n+1} \geq-(1+k) U_{j}^{n} .
$$

Thus (4.13)-(4.14) are true for $n$ replaced by $n+1$. Furthermore, we have

$$
\left|U_{j}^{n+1}\right| \geq(1+k)\left|U_{j}^{n}\right|
$$

and the result follows by induction. 
4.2. Existence of absorbing sets, attractors, and stabilization. We now set (4.1) in the context of dissipative dynamical systems. For fixed $h$ and $\Delta t$ let $S_{\Delta}^{h}: L_{2}^{h} \rightarrow L_{2}^{h}$ be defined by

$$
S_{\Delta}^{h}(\mathbf{V})=(I-\Delta t \gamma L) \mathbf{V}-\Delta t \mathbf{f}(\mathbf{V})
$$

The continuity of $S_{\Delta}^{h}$ follows directly from (F2); the equilibria of $S_{\Delta}^{h}$ are given by the set $\mathcal{E}^{h}$ of solutions to (3.8). In order for $S_{\Delta}^{h}$ to yield a dissipative dynamical system it is necessary to restrict the initial condition to the set

$$
K_{2}^{h}=\left\{\mathbf{V} \in L_{2}^{h}:|\mathbf{V}|_{\infty} \leq a\right\}
$$

for any $a \geq \bar{u}$. In the following we set

$$
K=\max _{|r| \leq a}\left\{\left|f^{\prime}(r)\right|\right\}
$$

We derive the following discrete analogues of (R1)-(R4) under appropriate restrictions on the timestep which are dependent upon initial data and $h$ and detailed in Theorem 4.10 .

$(\mathrm{RE} 1)^{h}$ For each $\mathbf{U}_{0} \in K_{2}^{h}$ there exists a unique solution of (4.1) $\mathbf{U}_{n} \in L_{2}^{h}$ for all $n>0$. The mapping $\mathbf{U}_{0} \rightarrow \mathbf{U}_{n}$ is continuous in $L_{2}^{h}$ for each $n>0$ and hence the family of solution operators $\left\{\left(S_{\Delta}^{h}\right)^{n}\right\}_{n \geq 0}$, defined by $\left(S_{\Delta}^{h}\right)^{n} \mathbf{U}_{0} \equiv \mathbf{U}_{n}$, forms a continuous semigroup on $K_{2}^{h}$.

$(\mathrm{RE} 2)^{h}$ There exist constants $\left\{\bar{\rho}_{i}\right\}_{i=1}^{2}$ independent of $h$ and $\Delta t$ such that the balls

$$
\begin{aligned}
& B_{1}^{h}=\left\{\mathbf{V} \in L_{2}^{h}:|\mathbf{V}| \leq \overline{\rho_{1}}\right\}, \\
& B_{2}^{h}=\left\{\mathbf{V} \in L_{2}^{h}:\|\mathbf{V}\| \leq \overline{\rho_{2}}\right\}
\end{aligned}
$$

are absorbing sets for the semigroup $\left\{\left(S_{\Delta}^{h}\right)^{n}\right\}_{n \geq 0}$, that is, for each $\mathbf{U}_{0} \in K_{2}^{h}$ there exists $\left\{n_{i}\right\}_{i=1}^{2}$ (depending on $\left\{\mathbf{U}_{0}, \rho_{1}\right\}$ and $\left\{\mathbf{U}_{0}, \rho_{2}\right\}$, respectively) such that

$$
\left(S_{\Delta}^{h}\right)^{n} \mathbf{U}_{0} \in B_{i}^{h} \quad \forall n \geq n_{i} \quad(i=1,2) .
$$

$(\mathrm{RE} 3)^{h}$ There exists a global attractor $\mathcal{A}^{h} \subset L_{2}^{h}$ for the semigroup $\left\{\left(S_{\Delta}^{h}\right)^{n}\right\}_{n \geq 0}$. Furthermore, $\mathcal{A}^{h}$ is connected and there exists a constant $\overline{\rho_{A}}$ independent of $h$ and $J$ such that

$$
\max \left\{|\mathbf{V}|,\|\mathbf{V}\|,|\mathbf{V}|_{\infty}\right\} \leq \overline{\rho_{A}} \quad \forall \mathbf{V} \in \mathcal{A}^{h}
$$

$(\mathrm{RE} 4)^{h}$ The functional defined by (3.9) is a Lyapunov functional for $\left\{\left(S_{\Delta}^{h}\right)^{n}\right\}_{t>0}$. In addition, for any $\mathbf{U}_{0} \in K_{2}^{h}$, the $\omega$-limit set is contained in $\mathcal{E}^{h}$.

We proceed by obtaining some estimates of $\mathbf{U}_{n}=\left(S_{\Delta}^{h}\right)^{n} \mathbf{U}_{0}$. We begin by proving a time discrete version of the maximum norm bounds of Lemma 2.1. The first bound is a special case of Theorem 3.3 in [12].

LEMмA 4.5. If the initial data and mesh parameters satisfy

$$
\mathbf{U}_{0} \in K_{2}^{h}
$$

and

$$
\Delta t \leq \frac{h^{2}}{\gamma C_{1}+K h^{2}}
$$


then

$$
\left|\mathbf{U}_{n}\right|_{\infty} \leq a \quad \forall n>0 .
$$

If, for some $\delta \in(0,2)$

$$
\Delta t \leq \min \left\{\frac{h^{2}}{\gamma C_{1}+K h^{2}}, \frac{(2-\delta) h^{2}}{\left(C_{0} \gamma\right)}\right\}
$$

and $a \geq m \geq \bar{u}$, then

$$
\left|\left(\mathbf{U}_{n}-m \mathbf{E}\right)_{+}\right|^{2} \leq\left(1+\Delta t \gamma \delta / C_{p}^{2}\right)^{-1}\left|\left(\mathbf{U}_{0}-m \mathbf{E}\right)_{+}\right|^{2}
$$

and

$$
\left|\left(\mathbf{U}_{n}+m \mathbf{E}\right)_{-}\right|^{2} \leq\left(1+\Delta t \gamma \delta / C_{p}^{2}\right)^{-1}\left|\left(\mathbf{U}_{0}+m \mathbf{E}\right)_{-}\right|^{2}
$$

Proof. We write (4.1) componentwise as

$$
U_{i}^{n+1}-a=U_{i}^{n}-a-\Delta t \gamma \sum_{j=1}^{J} L_{i j}\left(U_{j}^{n}-a\right)-\Delta t \gamma(L E)_{i} a-\Delta t f\left(U_{i}^{n}\right) .
$$

Let us assume that $\left|\mathbf{U}_{n}\right|_{\infty} \leq a$. Rewriting, we obtain $U_{i}^{n+1}-a=\left[1-\Delta t \gamma L_{i i}-\Delta t f^{\prime}\left(\xi_{i}^{n}\right)\right]\left(U_{i}^{n}-a\right)-\Delta t \gamma \sum_{j \neq i}^{J} L_{i j}\left(U_{j}^{n}-a\right)-\Delta t \gamma(L E)_{i} a-\Delta t f(a)$.

Since $\left|\xi_{j}^{n}\right| \leq a$ it follows from the fact that $f(a)>0$, the assumptions (3.5), (3.6) on $L$ and the restriction (4.15) on the timestep that

$$
U_{i}^{n+1}-a \leq 0
$$

and it follows that

$$
U_{i}^{n} \leq a
$$

by induction. Similarly it may be shown that

$$
U_{i}^{n} \geq-a
$$

For $a \geq m \geq \bar{u}$, it holds that

$$
\begin{aligned}
U_{i}^{n+1}-m & =\left(1-\Delta t \gamma L_{i i}\right)\left(U_{i}^{n}-m\right)-\Delta t \gamma \sum_{j \neq i}^{J} L_{i j}\left(U_{j}^{n}-m\right)-\Delta t \gamma(L E)_{i} m-\Delta t f\left(U_{i}^{n}\right) \\
& \leq\left(1-\Delta t \gamma L_{i i}\right)\left(U_{i}^{n}-m\right)-\Delta t \gamma \sum_{j \neq i}^{J} L_{i j}\left(U_{j}^{n}-m\right)-\Delta t f\left(U_{i}^{n}\right) \\
& \leq\left(1-\Delta t \gamma L_{i i}-\Delta t f^{\prime}\left(\xi_{i}^{n}\right)\right)\left(U_{i}^{n}-m\right)-\Delta t \gamma \sum_{j \neq i}^{J} L_{i j}\left(U_{j}^{n}-m\right)-\Delta t f(m),
\end{aligned}
$$


where $\left|\xi_{i}^{n}\right| \leq a$. Thus, if $U_{i}^{n} \leq m$, then by (4.15) and (3.5)

$$
\begin{aligned}
U_{i}^{n+1}-m & \leq\left(1-\Delta t \gamma L_{i i}-\Delta t f^{\prime}\left(\xi_{i}^{n}\right)\right)\left(U_{i}^{n}-m\right)_{+}-\Delta t \gamma \sum_{j \neq i} L_{i j}\left(U_{j}^{n}-m\right)_{+} \\
& =\left(U_{i}^{n}-m\right)_{+}-\Delta t \gamma \sum_{j} L_{i j}\left(U_{j}^{n}-m\right)_{+} .
\end{aligned}
$$

Alternatively, if $U_{i}^{n}>m$ then $f\left(U_{i}^{n}\right)>0$ and we again obtain

$$
U_{i}^{n+1}-m \leq\left(U_{i}^{n}-m\right)_{+}-\Delta t \gamma \sum_{j} L_{i j}\left(U_{j}^{n}-m\right)_{+} .
$$

Multiplying by $\left(U_{i}^{n+1}-m\right)_{+}$and summing gives

$$
\left\langle\mathbf{Z}_{n+1}-\mathbf{Z}_{n}, \mathbf{Z}_{n+1}\right\rangle+\Delta t \gamma\left\langle\mathbf{Z}_{n}, \mathbf{Z}_{n+1}\right\rangle_{A} \leq 0
$$

where

$$
\mathbf{Z}_{n}=\left(\mathbf{U}_{n}-m \mathbf{E}\right)_{+} \cdot
$$

This is the time discrete analogue of (3.16). Rearranging we obtain, by (3.2),

$$
\begin{aligned}
& \left|\mathbf{Z}_{n+1}\right|^{2}-\left|\mathbf{Z}_{n}\right|^{2}+\left|\mathbf{Z}_{n+1}-\mathbf{Z}_{n}\right|^{2}+2 \Delta t \gamma\left\|\mathbf{Z}_{n+1}\right\|^{2} \leq 2 \Delta t \gamma\left\|\mathbf{Z}_{n+1}-\mathbf{Z}_{n}\right\|\left\|\mathbf{Z}_{n+1}\right\| \\
& \quad \leq \frac{\gamma \Delta t}{2-\delta} \frac{C_{0}}{h^{2}}\left|\mathbf{Z}_{n+1}-\mathbf{Z}_{n}\right|^{2}+\gamma \Delta t(2-\delta)\left\|\mathbf{Z}_{n+1}\right\|^{2}
\end{aligned}
$$

and, provided that (4.16) holds, we obtain

$$
\left|\mathbf{Z}_{n+1}\right|^{2}\left(1+\delta \gamma \Delta t / C_{p}^{2}\right) \leq\left|\mathbf{Z}_{n}\right|^{2} .
$$

Similarly we estimate $\left(\mathbf{U}_{n}+m \mathbf{E}\right)_{-}$and obtain the desired results.

Remark. The preceding lemma is also true with the same proof if we set

$$
a=\max \left\{\left|\mathbf{U}_{0}\right|_{\infty}, \bar{u}\right\}
$$

LEMMA 4.6. If, for some $\delta \in(0,1)$

$$
\Delta t \leq \min \left\{2(1-\delta) \gamma h^{2} /\left(\gamma C_{0}^{\frac{1}{2}}+h K C_{p}\right)^{2}, h^{2} /\left(\gamma C_{1}+K h^{2}\right)\right\}
$$

then

$$
\left|\mathbf{U}_{n}\right|^{2} \leq\left|\mathbf{U}_{0}\right|^{2}\left(1+2 \Delta t \gamma \delta / C_{p}^{2}\right)^{-n}+\frac{C_{f} C(\Omega) C_{p}^{2}}{\gamma \delta}\left[1-\left(1+2 \Delta t \gamma \delta / C_{p}^{2}\right)^{-n}\right] .
$$

Proof. Taking the inner product of (4.1) with $\mathbf{U}_{n+1}$ yields

$$
\begin{aligned}
& \frac{1}{2} \partial\left|\mathbf{U}_{n+1}\right|^{2}+\frac{\Delta t}{2}\left|\partial \mathbf{U}_{n+1}\right|^{2}+\gamma\left\|\mathbf{U}_{n+1}\right\|^{2}+\left\langle\mathbf{f}\left(\mathbf{U}_{n+1}\right), \mathbf{U}_{n+1}\right\rangle \\
& \quad=\left\langle\mathbf{f}\left(\mathbf{U}_{n+1}\right)-\mathbf{f}\left(\mathbf{U}_{n}\right), \mathbf{U}_{n+1}\right\rangle+\gamma\left\langle\mathbf{U}_{n+1}-\mathbf{U}_{n}, \mathbf{U}_{n+1}\right\rangle_{A} \\
& \quad \leq K \Delta t\left|\partial \mathbf{U}_{n+1}\right|\left|\mathbf{U}_{n+1}\right|+\frac{\Delta t C_{0}^{\frac{1}{2}} \gamma}{h}\left|\partial \mathbf{U}_{n+1}\right|\left\|\mathbf{U}_{n+1}\right\| .
\end{aligned}
$$


Here we have used Lemma 4.5 and (3.2). Using Poincaré's inequality (3.1) and (3.12) we find that

$$
\begin{aligned}
& \frac{1}{2} \partial\left|\mathbf{U}_{n+1}\right|^{2}+\frac{\Delta t}{2}\left|\partial \mathbf{U}_{n+1}\right|^{2}+\gamma\left\|\mathbf{U}_{n+1}\right\|^{2} \\
& \quad \leq C_{f} C(\Omega)+\left(K \Delta t C_{p}+\frac{\Delta t C_{0}^{\frac{1}{2}} \gamma}{h}\right)\left|\partial \mathbf{U}_{n+1}\right|\left\|\mathbf{U}_{n+1}\right\|
\end{aligned}
$$

and completing the square on the left-hand side of this inequality yields

$$
\begin{aligned}
& \frac{1}{2} \partial\left|\mathbf{U}_{n+1}\right|^{2}+\sqrt{(2 \Delta t \gamma(1-\delta))\left|\partial \mathbf{U}_{n+1}\right|\left\|\mathbf{U}_{n+1}\right\|+\gamma \delta\left\|\mathbf{U}_{n+1}\right\|^{2}} \\
& \quad \leq C_{f} C(\Omega)+\left(K \Delta t C_{p}+\frac{\Delta t C_{0}^{\frac{1}{2}} \gamma}{h}\right)\left|\partial \mathbf{U}_{n+1}\right|\left\|\mathbf{U}_{n+1}\right\| .
\end{aligned}
$$

Under the timestep restriction (4.17) we have that

$$
\partial\left|\mathbf{U}_{n+1}\right|^{2}+2 \gamma \delta\left\|\mathbf{U}_{n+1}\right\|^{2} \leq 2 C_{f} C(\Omega)
$$

so that

$$
\left(1+2 \Delta t \gamma \delta / C_{p}^{2}\right)\left|\mathbf{U}_{n+1}\right|^{2} \leq\left|\mathbf{U}_{n}\right|^{2}+2 \Delta t C_{f} C(\Omega)
$$

and application of the standard discrete Gronwall lemma gives the result.

For any $\varepsilon>0$, let

$$
\rho_{1}=C_{f} C(\Omega) C_{p}^{2} /(\gamma \delta)+\varepsilon .
$$

It follows from Lemma 4.6 that there exists $n_{0}$ depending only on $\left|\mathbf{U}_{0}\right|$ and $\left|\mathbf{U}_{0}\right|_{\infty}$ such that

$$
\left|\mathbf{U}_{n}\right| \leq \rho_{1} \quad \forall n \geq n_{0}
$$

provided that (4.17) holds. Thus the following statement about absorbing sets has been established.

Proposition 4.7. There exists a constant $\rho_{1}$ independent of $h$ and $J$ such that the ball

$$
B_{1}^{h}=\left\{\mathbf{V} \in L_{2}^{h}:|\mathbf{V}| \leq \rho_{1}\right\}
$$

is absorbing in $\mathbf{U}^{h}$ under $S_{\Delta}^{h}$ provided the timestep restriction (4.17) holds.

We now prove the existence of an absorbing set in the $\|\bullet\|$ norm. To do this we employ a discrete version of the uniform Gronwall lemma, which is stated and proved in Appendix 2.

Proposition 4.8. There exists a constant $\rho_{2}$ independent of $h$ such that the ball

$$
B_{2}^{h}=\left\{\mathbf{V} \in L_{2}^{h}:\|\mathbf{V}\| \leq \rho_{2}\right\}
$$

is absorbing in $K_{2}^{h}$ under $S_{\Delta}^{h}$ provided the timestep restriction (4.17) and

$$
\Delta t \leq \min \left\{\frac{2 \gamma h^{2}}{\left(\gamma C_{0}^{\frac{1}{2}}+K C_{\rho} h\right)^{2}}, \frac{1-\delta_{1}}{2 C_{F}}\right\}
$$


holds for some $\delta_{1} \in(0,1)$.

Proof. Taking the $\langle\bullet, \bullet\rangle_{A}$ inner product of $M^{-1}$ times (4.1) with $\mathbf{U}_{n+1}$ gives

$$
\left\langle\mathbf{U}_{n+1}, \quad \partial \mathbf{U}_{n+1}\right\rangle_{A}+\gamma\left\langle M^{-1} A U_{n}, \quad d M^{-1} A U_{n+1}\right\rangle+\left\langle\mathbf{f}\left(\mathbf{U}_{n}\right), \quad \mathbf{U}_{n+1}\right\rangle_{A}=0 .
$$

Hence

$$
\begin{aligned}
& \frac{1}{2} \partial\left\|\mathbf{U}_{n+1}\right\|^{2}+\frac{\Delta t}{2}\left\|\partial \mathbf{U}_{n+1}\right\|^{2}+\gamma\left|M^{-1} A \mathbf{U}_{n+1}\right|^{2}+\left\langle\mathbf{f}\left(\mathbf{U}_{n+1}\right), \quad \mathbf{U}_{n+1}\right\rangle_{A} \\
& \quad=\gamma \Delta t\left\langle M^{-1} A \partial \mathbf{U}_{n+1}, M^{-1} A \mathbf{U}_{n+1}\right\rangle+\left\langle\mathbf{f}\left(\mathbf{U}_{n+1}\right)-\mathbf{f}\left(\mathbf{U}_{n}\right), \quad \mathbf{U}_{n+1}\right\rangle_{A} \\
& \quad \leq \gamma \Delta t\left|M^{-1} A \partial \mathbf{U}_{n+1}\left\|M^{-1} A \mathbf{U}_{n+1} \mid+K \Delta t\right\| \partial \mathbf{U}_{n+1}\|\| \mathbf{U}_{n+1} \| .\right.
\end{aligned}
$$

Using (3.3),(3.4) and splitting $\mathbf{f}$ as the difference of two monotone functions $f(u)=$ $f(u)+C_{F} u-C_{F} u$ and applying (3.7) we obtain

$$
\begin{aligned}
& \frac{1}{2} \partial\left\|\mathbf{U}_{n+1}\right\|^{2}+\frac{\Delta t}{2}\left\|\partial \mathbf{U}_{n+1}\right\|^{2}+\gamma\left|M^{-1} A \mathbf{U}_{n+1}\right|^{2} \\
& \quad \leq C_{F}\left\|\mathbf{U}_{n+1}\right\|^{2}+\frac{\gamma C_{0}^{\frac{1}{2}} \Delta t}{h}\left\|\partial \mathbf{U}_{n+1}\right\|\left|M^{-1} A \mathbf{U}_{n+1}\right|+K C_{p} \Delta t\left\|\partial \mathbf{U}_{n+1}\right\|\left|M^{-1} A \mathbf{U}_{n+1}\right| .
\end{aligned}
$$

Under restriction (4.19) we obtain

$$
\frac{1}{2} \partial\left\|\mathbf{U}_{n+1}\right\|^{2} \leq C_{F}\left\|\mathbf{U}_{n}\right\|^{2} .
$$

From equation (4.18) we have that

$$
2 \gamma \delta \sum_{n=K_{0}}^{N+K_{0}} \Delta t\left\|\mathbf{U}_{n+1}\right\|^{2} \leq 2 C_{f} C(\Omega) r+\left|\mathbf{U}_{K_{0}}\right|^{2}
$$

where

$$
r=\sum_{n=K_{0}}^{N+K_{0}} \Delta t=(N+1) \Delta t
$$

By taking $K_{0}$ sufficiently large and using Proposition 4.7 we obtain

$$
2 \gamma \delta \sum_{K_{0}}^{N+K_{0}} \Delta t\left\|\mathbf{U}_{n+1}\right\|^{2} \leq 2 C_{f} C(\Omega) r+\rho_{1}^{2} .
$$

Application of the discrete uniform Gronwall lemma to (4.20) (Appendix 2) gives

$$
\left\|\mathbf{U}_{N+K_{0}+1}\right\|^{2} \leq \exp \left(2 C_{F} r / \delta_{1}\right)\left[\frac{2 C_{f} C(\Omega) r+\rho_{1}^{2}}{2 \gamma r \delta}\right]
$$

for all $K_{0}$ sufficiently large. This completes the proof.

PROPOSITION 4.9. Let the mesh parameters satisfy

$$
\Delta t \leq \min \left\{\frac{h^{2}}{\gamma C_{1}+K h^{2}}, \frac{2(1-\delta) h^{2}}{\gamma C_{0}+K h^{2}}\right\}
$$

for some $\delta \in(0,1)$. Then $I^{h}(\bullet)$ is a Lyapunov functional for $S_{\Delta}^{h}$ on $K_{2}^{h}$. 
Proof. Since

$$
f(s)(r-s)=F(r)-F(s)-\frac{1}{2} f^{\prime}(\xi)(r-s)^{2}
$$

it follows that, after taking the inner product of (4.1) with $\partial \mathbf{U}_{n+1}$ and using Lemma 4.5 ,

$$
\Delta t\left|\partial \mathbf{U}_{n+1}\right|^{2}+I^{h}\left(\mathbf{U}_{n+1}\right) \leq I^{h}\left(\mathbf{U}_{n}\right)+\frac{\gamma \Delta t^{2}}{2}\left\|\partial \mathbf{U}_{n+1}\right\|^{2}+\frac{K \Delta t^{2}}{2}\left|\partial \mathbf{U}_{n+1}\right|^{2} .
$$

Therefore, using (3.2) and the timestep restriction (4.21) we obtain

$$
\delta \Delta t\left|\partial \mathbf{U}_{n+1}\right|^{2}+I^{h}\left(\mathbf{U}_{n+1}\right) \leq I^{h}\left(\mathbf{U}_{n}\right) .
$$

Clearly $I^{h}\left(\mathbf{U}_{n}\right)$ is nonincreasing in $n$ and if $I^{h}\left(\mathbf{U}_{n+1}\right)=I^{h}\left(\mathbf{U}_{n}\right)$, then $\left|\partial \mathbf{U}_{n+1}\right|=0$ by (4.22) which implies that $\mathbf{U}_{n+1}=\mathbf{U}_{n}$ and by (4.1) $\mathbf{U}_{n}=\mathbf{U}^{*} \in \mathcal{E}^{h}$. Thus $I^{h}(\bullet)$ is a Lyapunov functional for $S_{\Delta}^{h}(\bullet)$.

It follows from Lemma 2.1 and Propositions 2.3, 4.7, 4.8, 4.9 that the following result holds, noting that the boundedness of the attractor in $|\bullet|_{\infty}$ follows from Lemma 4.5 .

TheOREM 4.10. Setting

$$
\mathcal{A}^{h}=\omega\left(B_{1}^{h}\right),
$$

$(\mathrm{RE} 1)^{h}-(\mathrm{RE} 4)^{h}$ hold under restrictions (4.15), (4.16), (4.17), (4.19), and (4.21).

5. One step implicit approximations: existence of absorbing sets, attractors and stabilization. Given $\mathrm{U}_{n-1} \in L_{2}^{h}$ we consider the following schemes to find $\mathbf{U}_{n} \in L_{2}^{h}$ for $n \geq 1$ :

$$
M \partial \mathbf{U}_{n}+\frac{\gamma}{2} A\left(\mathbf{U}_{n}+\mathbf{U}_{n-1}\right)+M \hat{\mathbf{f}}\left(\mathbf{U}_{n}, \mathbf{U}_{n-1}\right)=0
$$

$$
M \partial \mathbf{U}_{n}+\gamma A \mathbf{U}_{n}+M \tilde{\mathbf{f}}\left(\mathbf{U}_{n}, \mathbf{U}_{n-1}\right)=0
$$

where

$$
\begin{array}{rcc}
\{\hat{\mathbf{f}}(\mathbf{U}, \mathbf{V})\}_{i}:= & \hat{f}\left(U_{i}, V_{i}\right), & \\
\hat{f}(a, b):= & (F(a)-F(b)) /(a-b) & a \neq b \\
\hat{f}(a, b):= & f(a) & a=b,
\end{array}
$$

and

$$
\begin{array}{cll}
\{\tilde{\mathbf{f}}(\mathbf{U}, \mathbf{V})\}_{i} & := & \mathbf{f}_{0}(\mathbf{U})-\mathbf{f}_{1}(\mathbf{V}) \\
f(r) & := & f_{0}(r)-f_{1}(r) .
\end{array}
$$

Under condition (F3) we may choose $f_{0}(\bullet)$ and $f_{1}(\bullet)$ to be monotone increasing; for example $f_{0}(r)=f(r)+r C_{F}, f_{1}(r)=r C_{F}$. We assume that such a choice has been made throughout this section. As in $\S 4$,

$$
K_{2}^{h}=\left\{\mathbf{V} \in L_{2}^{h}:|\mathbf{V}|_{\infty} \leq a\right\},
$$


where $a \geq \bar{u}$ and we set

$$
K=\max _{|r| \leq a}\left|f^{\prime}(r)\right|
$$

We also set

$$
K_{1}^{h} \equiv L_{2}^{h}
$$

for notational convenience.

Replacing $\mathbf{U}_{n-1}$ by $\mathbf{V}$ and $\mathbf{U}_{n}$ by $\mathbf{W}$ in each of (5.1)-(5.4) yields systems of algebraic equations for $\mathbf{W}$, given $\mathbf{V}$. Provided they have unique solution then we write $\mathbf{W}=S_{\Delta}^{h}(\mathbf{V})$. Under appropriate restrictions (see Theorems 5.6 and 5.10) we show below that schemes (S1) and (S2) satisfy $(\mathrm{RI} 1)^{h}-(\mathrm{RI} 4)^{h}$ with $j=1$ for (S1) and $j=2$ for (S2). We show that schemes (S3) and (S4) satisfy $(\mathrm{RI} 1)^{h},\left(\mathrm{RI}^{*}\right)^{h}$ and $(\mathrm{RI} 4)^{h}$ with $j=1$.

$(\mathrm{RI} 1)^{h}$ For each $\mathbf{U}_{0} \in K_{j}^{h}$ there exists a unique solution $\mathbf{U}_{n} \in L_{2}^{h}$ for all $n>0$. The mapping $\mathbf{U}_{0} \rightarrow \mathbf{U}_{n}$ is continuous in $L_{2}^{h}$ and hence the family of solution operators $\left\{\left(S_{\Delta}^{h}\right)^{n}\right\}_{n \geq 0}$, defined by $\left(S_{\Delta}^{h}\right)^{n} \mathbf{U}_{0} \equiv \mathbf{U}_{n}$, forms a continuous semigroup on $K_{j}^{h}$.

(RI2) ${ }^{h}$ There exist constants $\left\{\bar{\rho}_{i}\right\}_{i=1}^{2}$ independent of $h$ and $J$ such that the balls

$$
\begin{aligned}
& B_{1}^{h}=\left\{\mathbf{V} \in L_{2}^{h}:|\mathbf{V}| \leq \overline{\rho_{1}}\right\} \\
& B_{2}^{h}=\left\{\mathbf{V} \in L_{2}^{h}:\|\mathbf{V}\| \leq \overline{\rho_{2}}\right\}
\end{aligned}
$$

are absorbing sets for the semigroup $\left\{\left(S_{\Delta}^{h}\right)^{n}\right\}_{n \geq 0}$, that is, for each $\mathbf{U}_{0} \in K_{j}^{h}$ there exists $\left\{n_{i}\right\}_{i=1}^{2}$ (depending on $\left\{\mathbf{U}_{0}, \rho_{1}\right\}$ and $\left\{\mathbf{U}_{0}, \rho_{2}\right\}$, respectively) such that

$$
\left(S_{\Delta}^{h}\right)^{n} \mathbf{U}_{0} \in B_{i}^{h} \quad \forall n \geq n_{i} \quad(i=1,2) .
$$

$(\mathrm{RI} 3)^{h}$ There exists a global attractor $\mathcal{A}^{h} \subset L_{2}^{h}$ for the semigroup $\left\{\left(S_{\Delta}^{h}\right)^{n}\right\}_{n \geq 0}$. Furthermore $\mathcal{A}^{h}$ is connected and there exists a constant $\overline{\rho_{A}}$ independent of $h$ and $J$ such that

$$
\max \left\{|\mathbf{V}|,\|\mathbf{V}\|,|\mathbf{V}|_{\infty}\right\} \leq \overline{\rho_{A}} \quad \forall \mathbf{V} \in \mathcal{A}^{h} .
$$

$\left(\mathrm{RI}^{*}\right)^{h}$ Let $H^{h}$ be the normed vector space $\left\{\mathbb{R}^{J},\|\bullet\|\right\}$. Then $\left\{\left(S_{\Delta}^{h}\right)^{n}\right\}_{n \geq 0}$ is point dissipative on $H^{h}$ and there exists a connected global attractor $\mathcal{A}^{h} \subset L_{2}^{h}$ for the semigroup $\left\{\left(S_{\Delta}^{h}\right)^{n}\right\}_{n \geq 0}$.

$(\mathrm{RI} 4)^{h}$ The functional defined by (3.9) is a Lyapunov functional for $\left\{\left(S_{\Delta}^{h}\right)^{n}\right\}_{t>0}$. In addition, for any $\mathbf{U}_{0} \in K_{j}^{h}$, the $\omega$-limit set is contained in $\mathcal{E}^{h}$.

5.1. Schemes (S1) and (S2). We establish that (S1) and (S2) define dynamical systems and prove the existence of absorbing sets, a global attractor and a Lyapunov functional.

LEMMA 5.1. Suppose that the timestep restriction

$$
\Delta t \leq 1 / C_{F}
$$

holds for (S1) and the restriction

$$
\Delta t \leq 1 / K
$$

holds for (S2). Then (S1) and (S2) generate continuous solution operators $S_{\Delta}^{h}$ and unique sequences $\left\{\mathrm{U}_{n}\right\}_{n \geq 0}$ such that

$$
\left|\mathbf{U}_{n}\right|_{\infty} \leq a \quad \forall n \geq 0 .
$$


Furthermore, for $\bar{u} \leq m \leq a$ and any integer $k>1$,

$$
\left|\left(\mathbf{U}_{n+2 k}-m \mathbf{E}\right)_{+}\right| \leq\left(1+2 \gamma \Delta t / C_{p}^{2}\right)^{-k}\left|\left(\mathbf{U}_{n}-m \mathbf{E}\right)_{+}\right|
$$

and

$$
\left|\left(\mathbf{U}_{n+2 k}+m \mathbf{E}\right)_{-}\right| \leq\left(1+2 \gamma \Delta t / C_{p}^{2}\right)^{-k}\left|\left(\mathbf{U}_{n}+m \mathbf{E}\right)_{-}\right|
$$

Proof. First we show that (S1) generates a continuous operator $S_{\Delta}^{h}$ under the restriction (5.5). Existence of a solution follows from a well-known variational argument based on minimising the functional

$$
\frac{1}{2 \Delta t}|\mathbf{W}-\mathbf{V}|^{2}+\frac{\gamma}{2}\|\mathbf{W}\|^{2}+\langle\mathbf{F}(\mathbf{W}), \mathbf{E}\rangle
$$

over $L_{2}^{h}$ given $\mathbf{V} \in L_{2}^{h}$ (cf. [5]).

Let

$$
M \mathbf{W}^{i}+\Delta t \gamma A \mathbf{W}^{i}+\Delta t M \mathbf{f}\left(\mathbf{W}^{i}\right)=M \mathbf{V}^{i}, \quad i=1,2,
$$

and

$$
\mathbf{E}_{w}=\mathbf{W}^{1}-\mathbf{W}^{2}, \quad \mathbf{E}_{v}=\mathbf{V}^{1}-\mathbf{V}^{2}
$$

It follows that

$$
\left|\mathbf{E}_{w}\right|^{2}+\Delta t \gamma\left\|\mathbf{E}_{w}\right\|^{2}+\Delta t\left\langle\mathbf{f}\left(\mathbf{W}^{1}\right)-\mathbf{f}\left(\mathbf{W}^{2}\right), \mathbf{E}_{w}\right\rangle=\left\langle\mathbf{E}_{v}, \mathbf{E}_{w}\right\rangle .
$$

Using

$$
\left\langle\mathbf{f}\left(\mathbf{W}^{1}\right)-\mathbf{f}\left(\mathbf{W}^{2}\right), \mathbf{E}_{w}\right\rangle \geq-C_{F}\left|\mathbf{E}_{w}\right|^{2}
$$

and (5.5) we have

$$
\Delta t \gamma\left\|\mathbf{E}_{w}\right\|^{2} \leq\left|\mathbf{E}_{v} \| \mathbf{E}_{w}\right| .
$$

Hence, under the restriction (5.5) the scheme (S1) generates a unique sequence $\left\{\mathrm{U}_{n}\right\}$ and defines a continuous solution operator $S_{\Delta}^{h}$. We now turn to the proof of (5.7)-(5.9) for (S1).

For $n \geq 0$ set $\mathbf{Z}_{n}=\mathbf{U}_{n}-m \mathbf{E}$. It follows that

$$
\left\langle\partial \mathbf{Z}_{n},\left(\mathbf{Z}_{n}\right)_{+}\right\rangle+\gamma\left\langle\mathbf{Z}_{n},\left(\mathbf{Z}_{n}\right)_{+}\right\rangle_{A}+\left\langle\mathbf{f}\left(\mathbf{U}_{n}\right),\left(\mathbf{Z}_{n}\right)_{+}\right\rangle=-m\left\langle\mathbf{E},\left(\mathbf{Z}_{n}\right)_{+}\right\rangle_{A} .
$$

Noting (F2), (3.6), (3.7) and the inequality

$$
(b-c) b_{+} \geq\left(\left|b_{+}\right|^{2}-\left|c_{+}\right|^{2}\right) / 2 \quad \forall b, c \in \mathbb{R},
$$

we have

$$
\frac{1}{2} \partial\left|\left(\mathbf{Z}_{n}\right)_{+}\right|^{2}+\gamma\left|\left(\mathbf{Z}_{n}\right)_{+}\right|^{2} / C_{p}^{2} \leq 0
$$

or

$$
\left(1+2 \Delta t \gamma / C_{p}^{2}\right)\left|\left(\mathbf{Z}_{n}\right)_{+}\right|^{2} \leq\left|\left(\mathbf{Z}_{n-1}\right)_{+}\right|^{2}
$$


and (5.8) is an immediate consequence. Equation (5.9) is proved similarly by consideration of $\mathbf{Z}_{n}=\mathbf{U}_{n}+m \mathbf{E}$ and then (5.7) is a consequence of (5.8) and (5.9) thus completing the proof of the Lemma for (S1).

Since $M+\Delta t A$ is positive definite the system of equations (5.2) always has a unique solution so that (S2) trivially generates a unique sequence $\left\{\mathbf{U}_{n}\right\}$ and the continuity of $f$ implies that $S_{\Delta}^{h}$ is continuous because of the finite dimensionality of $L_{2}^{h}$. Again, introducing $\mathbf{Z}_{n}=\mathbf{U}_{n}-m \mathbf{E}$ it follows from (3.6) that

$$
\left\langle\partial \mathbf{Z}_{n},\left(\mathbf{Z}_{n}\right)_{+}\right\rangle+\gamma\left\langle\mathbf{Z}_{n},\left(\mathbf{Z}_{n}\right)_{+}\right\rangle_{A}+\left\langle\mathbf{f}\left(\mathbf{U}_{n-1}\right),\left(\mathbf{Z}_{n}\right)_{+}\right\rangle \leq 0
$$

Either

$$
Z_{i}^{n-1}=U_{i}^{n-1}-m \geq 0
$$

and so

$$
\left(-Z_{i}^{n-1}+\Delta t f\left(U_{i}^{n-1}\right)\right)\left(U_{i}^{n}-m\right)_{+} \geq-\left(Z_{i}^{n-1}\right)\left(Z_{i}^{n}\right)_{+}=-\left(Z_{i}^{n-1}\right)_{+}\left(Z_{i}^{n}\right)_{+}
$$

or

$$
Z_{i}^{n-1}=U_{i}^{n-1}-m<0
$$

and

$$
\begin{aligned}
& \left(-Z_{i}^{n-1}+\Delta t f\left(U_{i}^{n-1}\right)\right)\left(U_{i}^{n}-m\right)_{+} \\
& \quad \geq\left(-Z_{i}^{n-1}+\Delta t\left(f\left(U_{i}^{n-1}\right)-f(m)\right)\right)\left(U_{i}^{n}-m\right)_{+} \\
& \quad=\left(-1+\Delta t f^{\prime}(\xi)\right) Z_{i}^{n-1}\left(Z_{i}^{n}\right)_{+} \geq 0=-\left(Z_{i}^{n-1}\right)_{+}\left(Z_{i}^{n}\right)_{+}
\end{aligned}
$$

provided

$$
\left\|\mathbf{U}_{n-1}\right\| \leq a
$$

since (5.6) holds and $f(m) \geq 0$. Thus we have shown that

$$
\Delta t\left\langle\mathbf{f}\left(\mathbf{U}_{n-1}\right),\left(\mathbf{Z}_{n}\right)_{+}\right\rangle \geq-\left\langle\left(\mathbf{Z}_{n-1}\right)_{+},\left(\mathbf{Z}_{n}\right)_{+}\right\rangle+\left\langle\mathbf{Z}_{n-1},\left(\mathbf{Z}_{n}\right)_{+}\right\rangle .
$$

Hence (5.10) follows from (5.11) provided (5.12) holds. By induction we deduce that (5.7) holds and then (5.8) and (5.9) by arguments similar to those used for (S1).

Remark. As for the explicit scheme, the preceding lemma is also true (with an identical proof) if $a=\max \left\{\left|\mathbf{U}_{0}\right|_{\infty}, \bar{u}\right\}$.

From Lemma 5.1 we have the following.

Proposition 5.2. For each $\mathbf{U}_{0} \in K_{1}^{h}$ (respectively, $K_{2}^{h}$ ) there exists a unique solution of (5.1) (respectively, (5.2)) $\mathbf{U}_{n} \in L_{2}^{h}$ provided (5.5) holds (respectively, (5.6)). The mapping $\mathbf{U}_{0} \rightarrow \mathbf{U}_{n}$ is continuous in $L_{2}^{h}$ for each $n>0$ and hence the family of solution operators $\left\{\left(S_{\Delta}^{h}\right)^{n}\right\}_{n \geq 0}$ defined by $\left(S_{\Delta}^{h}\right)^{n} \mathbf{U}_{0}=\mathbf{U}_{n}$ forms a discrete continuous semigroup on $K_{1}^{h}$ (respectively, $K_{2}^{h}$ ).

LEMMA 5.3. For schemes (S1) and (S2)

$$
\left|\mathbf{U}_{n}\right|^{2} \leq\left|\mathbf{U}_{0}\right|^{2}\left(1+2 \Delta t \gamma \delta / C_{p}^{2}\right)^{-n}+\frac{C_{f} C(\Omega) C_{p}^{2}}{\gamma \delta}\left[1-\left(1+2 \Delta t \gamma \delta / C_{p}^{2}\right)^{-n}\right]
$$

where $\delta=1$ for $(\mathrm{S} 1)$ and $\delta \in(0,1)$ for $(\mathrm{S} 2)$, provided that the timestep restriction

$$
\Delta t \leq \min \left\{1 / K, 2 \gamma(1-\delta) /\left(K^{2} C_{p}^{2}\right)\right\}
$$


holds for (S2).

Proof. Taking the inner product of (5.1) with $\mathbf{U}_{n}$ and using (3.1) yields

$$
\begin{aligned}
& \frac{1}{2} \partial\left|\mathbf{U}_{n}\right|^{2}+\frac{1}{2} \Delta t\left|\partial \mathbf{U}_{n}\right|^{2}+\gamma\left\|\mathbf{U}_{n}\right\|^{2} \leq C_{f} C(\Omega) \\
& \quad \Rightarrow \frac{1}{2} \partial\left|\mathbf{U}_{n}\right|^{2}+\frac{1}{2} \Delta t\left|\partial \mathbf{U}_{n}\right|^{2}+\gamma\left|\mathbf{U}_{n}\right|^{2} / C_{p}^{2} \leq C_{f} C(\Omega),
\end{aligned}
$$

where we have used (3.12) and Poincaré's inequality. The bound (5.13) follows from application of Gronwall's inequality. Similarly for (S2) we find, using (5.7),

$$
\frac{1}{2} \partial\left|\mathbf{U}_{n}\right|^{2}+\frac{1}{2} \Delta t\left|\partial \mathbf{U}_{n}\right|^{2}+\gamma\left\|\mathbf{U}_{n}\right\|^{2} \leq C_{f} C(\Omega)+K \Delta t\left|\partial \mathbf{U}_{n} \| \mathbf{U}_{n}\right|
$$

so that

$$
\frac{1}{2} \partial\left|\mathbf{U}_{n}\right|^{2}+\frac{1}{2} \Delta t\left|\partial \mathbf{U}_{n}\right|^{2}+\gamma\left|\mathbf{U}_{n}\right|^{2} / C_{p}^{2} \leq C_{f} C(\Omega)+K \Delta t\left|\partial \mathbf{U}_{n}\right|\left|\mathbf{U}_{n}\right|
$$

and under the timestep restriction, completing the square on the left-hand side it follows that

$$
\frac{1}{2} \partial\left|\mathbf{U}_{n}\right|^{2}+\delta \gamma\left|\mathbf{U}_{n}\right|^{2} / C_{o}^{2} \leq C_{f} C(\Omega)
$$

Applying the Gronwall inequality, the proof of the lemma is complete.

We may now prove the following.

Proposition 5.4. For scheme (S1) (respectively, (S2)) there exist constants $\rho_{1}, \rho_{2}$ independent of $h$ such that the balls

$$
B_{1}^{h}=\left\{\mathbf{V} \in L_{2}^{h}:|\mathbf{V}| \leq \rho_{1}\right\}, \quad B_{2}^{h}=\left\{\mathbf{V} \in L_{2}^{h}:\|\mathbf{V}\| \leq \rho_{2}\right\}
$$

are absorbing in $K_{1}^{h}$ (respectively, $K_{2}^{h}$ ) for $S_{\Delta}^{h}$ provided the timestep restriction

$$
\Delta t \leq \frac{1-\delta}{2 C_{F}}, \quad \text { some } \delta \in(0,1),
$$

holds (respectively,

$$
\left.\Delta t \leq \min \left\{\frac{1}{K}, \frac{1-\delta_{1}}{2 C_{F}}, \frac{2 \gamma\left(1-\delta_{2}\right)}{K^{2} C_{p}^{2}}\right\}, \text { some } \delta_{i} \in(0,1)\right) .
$$

Proof. The absorbing sets in $|\bullet|$ follow from Lemma 5.3. Taking the $\langle\bullet, \bullet\rangle_{A}$ inner product of $M^{-1}$ times (5.1) with $\partial \mathbf{U}_{n}$ yields, using the monotonicity of $f(u)+C_{F} u$

$$
\left\|\mathbf{U}_{n+1}\right\|^{2}-\left\|\mathbf{U}_{n}\right\|^{2} \leq 2 \Delta t C_{F}\left\|\mathbf{U}_{n+1}\right\|^{2} .
$$

By (5.15) we have

$$
2 \Delta t \gamma \sum_{n=K_{0}}^{N+K_{0}}\left\|\mathbf{U}_{n+1}\right\|^{2} \leq 2 r C_{f} C(\Omega)+\left|\mathbf{U}_{K_{0}}\right|^{2},
$$

where $r=(N+1) \Delta t$. Hence, for $K_{0}$ sufficiently large, Lemma 5.3 gives

$$
\sum_{n=K_{0}}^{N+K_{0}} \Delta t\left\|\mathbf{U}_{n+1}\right\|^{2} \leq \frac{C_{f} C(\Omega) r}{\gamma}+\frac{\rho_{1}^{2}}{2 \gamma} .
$$


Applying the discrete uniform Gronwall Lemma (Appendix 2) we obtain

$$
\left\|\mathbf{U}_{N+K_{0}+1}\right\|^{2} \leq \exp \left(\frac{2 C_{F} r}{\delta}\right)\left[\frac{C_{f} C(\Omega)}{\gamma}+\frac{\rho_{1}^{2}}{2 r \gamma}\right]
$$

for arbitrarily large $K_{0}$. The statement of the lemma follows for (S1).

For (S2), a similar analysis as for (S1) yields

$$
\begin{aligned}
& \frac{1}{2} \partial\left\|\mathbf{U}_{n}\right\|^{2}+\frac{\Delta t}{2}\left\|\partial \mathbf{U}_{n}\right\|^{2}+\gamma\left|M^{-1} A \mathbf{U}_{n}\right|^{2}+\left\langle\mathbf{f}\left(\mathbf{U}_{n}\right), \mathbf{U}_{n}\right\rangle_{A}=\left\langle\mathbf{f}\left(\mathbf{U}_{n}\right)-\mathbf{f}\left(\mathbf{U}_{n-1}\right), \mathbf{U}_{n}\right\rangle_{A} \\
& \quad=\left\langle\mathbf{f}\left(\mathbf{U}_{n}\right)-\mathbf{f}\left(\mathbf{U}_{n-1}\right), M^{-1} A \mathbf{U}_{n}\right\rangle \leq K \Delta t\left|\partial \mathbf{U}_{n} \| M^{-1} A \mathbf{U}_{n}\right| \\
& \quad \leq K \Delta t C_{p}\left\|\partial \mathbf{U}_{n}\right\|\left|M^{-1} A \mathbf{U}_{n}\right|
\end{aligned}
$$

using inequality (3.3). Using the monotonicity of $f(u)+C_{F} u$ we obtain, under restriction (5.18),

$$
\frac{1}{2} \partial\left\|\mathbf{U}_{n}\right\|^{2} \leq C_{F}\left\|\mathbf{U}_{n}\right\|^{2}
$$

From (5.16) we have

$$
\frac{1}{2} \partial\left|\mathbf{U}_{n}\right|^{2}+\frac{\Delta t}{2}\left|\partial \mathbf{U}_{n}\right|^{2}+\frac{\gamma(1-\delta)}{C_{p}^{2}}\left|\mathbf{U}_{n}\right|^{2}+\frac{\gamma}{\delta}\left\|\mathbf{U}_{n}\right\|^{2} \leq C_{f} C(\Omega)+K \Delta t\left|\partial \mathbf{U}_{n} \| \mathbf{U}_{n}\right|
$$

Completing the square on the left-hand side under restriction (5.18) we obtain

$$
\frac{1}{2} \partial\left|\mathbf{U}_{n}\right|^{2}+\gamma \delta_{2}\left\|\mathbf{U}_{n}\right\|^{2} \leq C_{f} C(\Omega)
$$

and the argument now proceeds as for (S1) to obtain

$$
\left\|\mathbf{U}_{N+K_{0}+1}\right\|^{2} \leq \exp \left(\frac{2 C_{F} r}{\delta}\right)\left[\frac{C_{f} C(\Omega)}{\gamma \delta_{2}}+\frac{\rho_{1}^{2}}{2 r \gamma \delta_{2}}\right]
$$

for arbitrarily large $K_{0}$. The statement of the lemma follows for (S2).

Proposition 5.5. Suppose that the timestep restrictions (5.5) (respectively, (5.6)) hold for (S1) (respectively, (S2)). Then for (S1) (respectively, (S2)) $I^{h}(\bullet)$ is a Lyapunov functional for $S_{\Delta}^{h}$ on $K_{1}^{h}$ (respectively, $\left.K_{2}^{h}\right)$.

Proof. Taking the inner product of (5.1) with $\partial \mathbf{U}_{n}$ yields

$$
\Delta t\left|\partial \mathbf{U}_{n}\right|^{2}+\frac{\gamma}{2}\left[\left\|\mathbf{U}_{n}\right\|^{2}-\left\|\mathbf{U}_{n-1}\right\|^{2}+\left\|\mathbf{U}_{n}-\mathbf{U}_{n-1}\right\|^{2}\right]+\left\langle\mathbf{f}\left(\mathbf{U}_{n}\right), \mathbf{U}_{n}-\mathbf{U}_{n-1}\right\rangle=0
$$

Note that

$$
f(r)(r-s)=F(r)-F(s)+\frac{f^{\prime}(\xi)}{2}(r-s)^{2}
$$

Hence for (S1),

$$
\left|\partial \mathbf{U}_{n}\right|^{2}+\frac{\Delta t \gamma}{2}\left\|\partial \mathbf{U}_{n}\right\|^{2}+\partial I^{h}\left(\mathbf{U}_{n}\right) \leq \frac{C_{F} \Delta t}{2}\left|\partial \mathbf{U}_{n}\right|^{2}
$$

Under the restriction (5.5) $S_{\Delta}^{h}$ defines a dynamical system and, in addition,

$$
\partial I^{h}\left(\mathbf{U}_{n}\right) \leq-\frac{1}{2}\left|\partial \mathbf{U}_{2}\right|^{2}
$$


and the statament of the theorem follows.

An identical argument holds for (S2) if we note that

$$
f(r)(r-s) \geq F(r)-F(s)-\frac{K}{2}(r-s)^{2}
$$

for $|r|,|s| \leq a$.

By Lemma 2.1 and Propositions 2.3, 5.2, and 5.5 we have proved the following.

THEOREM 5.6. Let $\mathcal{A}=\omega\left(B_{1}^{h}\right)$ for $(\mathrm{S} 1)$ and (S2). Then, under the restrictions (5.5), (5.17) for (S1) and (5.6), (5.18) for (S2) (RI1) ${ }^{h}-(\mathrm{RI} 4)^{h}$ hold with $j=1$ for (S1) and $j=2$ for $(\mathrm{S} 2)$.

5.2. Schemes (S3) and (S4). We establish that (S3) and (S4) define dynamical systems on $L_{2}^{h}$ and prove the existence of a global attractor by establishing a Lyapunov functional structure. We note that the proof of uniqueness can be used to prove continuity of $S_{\Delta}^{h}$ as for the scheme (S1).

LEMMA 5.7. There exist unique sequences generated by (S3) and (S4) for any $\mathbf{U}_{0} \in L_{2}^{h}$ provided, in the case of (S3), that for some $\delta \in(0,1)$,

$$
\Delta t \leq \max \left\{\frac{8 \gamma(1-\delta)}{\left(C_{F} C_{p}\right)^{2}}, \frac{2}{C_{F}}\right\} .
$$

Proof. We consider (S3) first. The analysis of existence and uniqueness is identical to that used by [5] for a similar scheme applied to the Cahn-Hilliard equation. Set

$$
\left\{\mathbf{F}^{n}(\mathbf{W})\right\}_{i}=\int_{0}^{W_{i}} \hat{f}\left(U_{i}^{n}, s\right) d s+c_{i},
$$

where $c_{i}$ is chosen so that $\left\{\mathbf{F}^{n}(\mathbf{W})\right\}_{i} \geq 0$. A standard argument in the calculus of variations yields a minimiser of the functional

$$
\frac{1}{2 \Delta t}\left|\mathbf{W}-\mathbf{U}_{n-1}\right|^{2}+\frac{\gamma}{4}\|\mathbf{W}\|^{2}+\frac{\gamma}{2}\left\langle\mathbf{U}_{n-1}, \mathbf{W}\right\rangle_{A}+\left\langle\mathbf{E}, \mathbf{F}^{n}(\mathbf{W})\right\rangle
$$

which satisfies the Euler-Lagrange equations (5.3). Furthermore, from [5],

$$
\left(\hat{f}\left(r, s_{1}\right)-\hat{f}\left(r, s_{2}\right)\right)\left(s_{1}-s_{2}\right)=F\left[s_{1}, r, s_{2}\right]\left(s_{1}-s_{2}\right)^{2} \geq-C_{F}\left(s_{1}-s_{2}\right)^{2} / 2,
$$

where $F\left[s_{1}, r, s_{2}\right]$ denotes the second divided difference. Thus, denoting the difference of two solutions to (5.3) by $\mathbf{Y}$ for given $\mathbf{U}_{n-1}$ we obtain

$$
|\mathbf{Y}|^{2}+\frac{\Delta t \gamma}{2}\|\mathbf{Y}\|^{2} \leq \Delta t C_{F}|\mathbf{Y}|^{2} / 2 \leq \Delta t C_{F} C_{p}\|\mathbf{Y}\||\mathbf{Y}| / 2
$$

Uniqueness under the condition (5.19) on $\Delta t$ follows.

Because of $(\mathrm{F} 3), f_{0}(\bullet)$ is monotone increasing and the existence and uniqueness of solutions to (5.4) is an immediate consequence of the method of monotonicity.

Thus we have established the following.

Proposition 5.8. For each $\mathbf{U}_{0} \in L_{2}^{h}$ there exists a unique solution of (5.3),(5.4) $\mathbf{U}_{n} \in L_{2}^{h}$ provided (5.19) holds for (S3). The mapping $\mathbf{U}_{0} \rightarrow \mathbf{U}_{n}$ is continuous in $L_{2}^{h}$ for each $n>0$ and hence the family of solution operators $\left\{\left(S_{\Delta}^{h}\right)^{n}\right\}_{n \geq 0}$ defined by $\left(S_{\Delta}^{h}\right)^{n} \mathbf{U}_{0}=\mathbf{U}_{n}$ forms a discrete continuous semigroup on $L_{2}^{h}$. 
PROPOSITION 5.9. Under the restriction (5.19) for scheme (S3) and no restriction for (S4), $I^{h}(\bullet)$ is a Lyapunov functional on $L_{2}^{h}$ for the dynamical systems generated by schemes (S3) and (S4).

Proof. Taking the inner product of (S3) with $\Delta t \partial \mathbf{U}_{n}$ yields

$$
\Delta t\left|\partial \mathbf{U}_{n}\right|^{2}+I^{h}\left(\mathbf{U}_{n}\right)-I^{h}\left(\mathbf{U}_{n-1}\right)=0
$$

This is the discrete analogue of (3.18) and the conclusion of the lemma is evident for (S3). For (S4) we use the inequality

$$
\begin{aligned}
& \left(f_{0}(r)-f_{1}(s)\right)(r-s) \\
& \quad=F_{0}(r)-F_{0}(s)+\frac{f_{0}^{\prime}(\xi)}{2}(r-s)^{2}+F_{1}(s)-F_{1}(r)+\frac{f_{1}^{\prime}(\eta)}{2}(r-s)^{2} \geq F(r)-F(s),
\end{aligned}
$$

which holds as a result of (F3) and the monotonicity of $f_{0}$ and $f_{1}$. Taking the inner product of (5.4) with $\Delta t \partial \mathbf{U}_{n}$, yields

$$
\Delta t\left|\partial \mathbf{U}_{n}\right|^{2}+I^{h}\left(\mathbf{U}_{n}\right)-I^{h}\left(\mathbf{U}_{n-1}\right)+\frac{\gamma \Delta t^{2}}{2}\left\|\partial \mathbf{U}_{n}\right\|^{2} \leq 0
$$

and the conclusion follows for (S4).

Applying Lemmas 2.1 and 2.4 and Propositions 2.5, 5.8, and 5.9 we establish the following

THEOREM 5.10. Then, under the restriction (5.19) for (S3) and no restriction for $(\mathrm{S} 4),(\mathrm{RI} 1)^{h},\left(\mathrm{RI}^{*}\right)^{h}$ and (RI4) ${ }^{h}$ hold with $j=1$.

6. Multistep backward differentiation methods. In this section we consider backward differentiation formulae. Given $\left\{\mathbf{U}_{j} \in L_{2}^{h}, j=0,1, \ldots, q-1\right\}$ we define the sequence $\left\{\mathbf{U}_{n} \in L_{2}^{h}\right\}$ by

$$
M\left(\sum_{j=1}^{q} \frac{\Delta t^{j-1}}{j} \partial^{j} \mathbf{U}_{n}\right)+\gamma A \mathbf{U}_{n}+M \mathbf{f}\left(\mathbf{U}_{n}\right)=0
$$

where $q \geq 1$ is a given integer and $\partial^{j}$ is defined recursively by

$$
\partial^{j} \mathbf{V}_{n}:=\frac{\partial^{j-1} \mathbf{V}_{n}-\partial^{j-1} \mathbf{V}_{n-1}}{\Delta t}, \quad j \geq 1
$$

This scheme is the result of applying the $q$-step backward differentiation formula to (1.8). For $q \in[1,6]$ the formula is known to be $A(\alpha)$-stable [18]. Since in order to find $\mathbf{U}_{n}$ one solves a system of the form

$$
\left(\alpha_{q} M+\Delta t \gamma A\right) \mathbf{W}+\Delta t M \mathbf{f}(\mathbf{W})=\mathbf{b}
$$

it is straightforward to apply the proof of Lemma 5.1 to show the following.

LEMMA 6.1. Suppose that the timestep restriction

$$
\Delta t \leq \alpha_{q} / C_{F}
$$

holds. Then there exists a unique sequence $\left\{\mathbf{U}_{n}\right\}$ satisfying equation (6.1) for given $\left\{\mathbf{U}_{j}\right\}_{0}^{q-1}$. 
Since knowing $\left\{\partial^{j} \mathbf{U}_{n}\right\}_{j=0}^{q-1}$ is equivalent to knowing $\left\{\mathbf{U}_{n-j}\right\}_{j=0}^{q-1}$ the system (6.1) is equivalent to

$$
M\left(\sum_{j=0}^{q-1} \frac{\Delta t^{j}}{j+1} \partial^{j} \mathbf{U}_{n}\right)+\Delta t \gamma A \mathbf{U}_{n}+\Delta t M \mathbf{f}\left(\mathbf{U}_{n}\right)=M\left(\sum_{j=0}^{q-1} \frac{\Delta t^{j}}{j+1} \partial^{j} \mathbf{U}_{n-1}\right)
$$

$$
\Delta t \partial^{j} \mathbf{U}_{n}-\partial^{j-1} \mathbf{U}_{n}=-\partial^{j-1} \mathbf{U}_{n-1}, \quad j=1,2, \ldots, q-1 .
$$

We let $\hat{\mathbf{V}}=\left(\mathbf{V}^{(1)}, \ldots, \mathbf{V}^{(q)}\right)$ be an arbitrary element of $\hat{L}_{2}^{h}:=\left(L_{2}^{h}\right)^{q}$ and set

$$
\hat{\mathbf{U}}_{n}:=\left(\mathbf{U}_{n}, \Delta t \partial \mathbf{U}_{n}, \ldots, \Delta t^{q-1} \partial^{q-1} \mathbf{U}_{n}\right) .
$$

It follows that, under the conditions of Lemma 6.1, there exists a continuous mapping $\hat{S}_{\Delta}^{h}: \hat{L}_{2}^{h} \rightarrow \hat{L}_{2}^{h}$ defined by

$$
\hat{\mathbf{W}}:=\hat{S}_{\Delta}^{h}(\hat{\mathbf{V}})
$$

where

$$
\begin{gathered}
M\left(\sum_{j=1}^{q} \frac{\mathbf{W}^{(j)}}{j}\right)+\Delta t \gamma A \mathbf{W}^{(1)}+\Delta t M \mathbf{f}\left(\mathbf{W}^{(1)}\right)=M\left(\sum_{j=1}^{q} \frac{\mathbf{V}^{(j)}}{j}\right) \\
\mathbf{W}^{(j)}-\mathbf{W}^{(j-1)}=-\mathbf{V}^{(j-1)}, \quad j=2, \ldots, q
\end{gathered}
$$

and

$$
\hat{\mathbf{U}}_{n}=\hat{S}_{\Delta}^{h}\left(\hat{\mathbf{U}}_{n-1}\right), \quad n \geq 1 .
$$

Thus (6.1) defines a discrete dynamical system on $\hat{L}_{2}^{h}$ via (6.7). We now proceed to show the properties of the dynamical system. Clearly a fixed point $\hat{\mathbf{U}}^{*}$ of $\hat{S}_{\Delta}^{h}$ satisfies

$$
\gamma A \mathbf{U}^{*(1)}+M \mathbf{f}\left(\mathbf{U}^{*(1)}\right)=0, \quad \mathbf{U}^{*(j)}=0 j=2, \ldots, q .
$$

It follows that the set of equilibria $\hat{\mathcal{E}}^{h}$ is given by $\hat{\mathbf{U}}^{*}=\left(\mathbf{U}^{*}, 0,0, \ldots, 0\right)$ where $\mathbf{U}^{*} \in \mathcal{E}^{h}$ is defined by (3.8). Thus $\hat{\mathcal{E}}^{h}$ is bounded and it follows that, by Proposition 2.5, the existence of a Lyapunov functional is sufficient to ensure the existence of a global attractor. In the case $q=1$ we set

$$
\hat{I}_{1}(\bullet)=I^{h}(\bullet) .
$$

For $q=2,3$ we set

$$
\hat{I}_{2}(\hat{\mathbf{V}})=\frac{1}{4 \Delta t}\left|\mathbf{V}^{(2)}\right|^{2}+I^{h}\left(\mathbf{V}^{(1)}\right)
$$

and

$$
\hat{I}_{3}(\hat{\mathbf{V}})=\frac{1}{6 \Delta t}\left|\mathbf{V}^{(3)}\right|^{2}+\frac{5}{12 \Delta t}\left|\mathbf{V}^{(2)}\right|^{2}+I^{h}\left(\mathbf{V}^{(1)}\right)
$$


We introduce the following norms on $\hat{L}_{2}^{h}$ :

$$
|\hat{\mathbf{V}}|=\left(\sum_{j=1}^{q}\left|\mathbf{V}^{(j)}\right|^{2}\right)^{\frac{1}{2}}
$$

and

$$
\|\hat{\mathbf{V}}\|=\left(\sum_{j=1}^{q}\left\|\mathbf{V}^{(j)}\right\|^{2}\right)^{\frac{1}{2}}
$$

It is clear that $I(i)$ of $\S 2$ is satisfied. Taking the inner product of (6.1) with $\Delta t \partial \mathbf{U}_{n}$ yields

$$
\begin{gathered}
\sum_{j=1}^{q} \frac{\Delta t^{j}}{j}\left\langle\partial^{j} \mathbf{U}_{n}, \partial \mathbf{U}_{n}\right\rangle+\frac{\gamma}{2} \Delta t^{2}\left\|\partial \mathbf{U}_{n}\right\|^{2}+I^{h}\left(\mathbf{U}_{n}\right) \\
\leq I^{h}\left(\mathbf{U}_{n-1}\right)+\frac{C_{F} \Delta t^{2}}{2}\left|\partial \mathbf{U}_{n}\right|^{2},
\end{gathered}
$$

where we have used the argument of Proposition 5.5. It remains to evaluate the first term on the left-hand side of (6.11) which we call $\Gamma_{q} / \Delta t$. We set $\mathbf{Z}_{n}=\mathbf{U}_{n}-\mathbf{U}_{n-1}$ and consider the cases $q=2$ and $q=3$ in turn.

$\mathbf{q}=2$. It may be shown that

$$
\begin{aligned}
\Gamma_{2} & =\left\langle\mathbf{U}_{n}-\mathbf{U}_{n-1}+\frac{1}{2}\left(\mathbf{U}_{n}-2 \mathbf{U}_{n-1}+\mathbf{U}_{n-2}\right), \mathbf{U}_{n}-\mathbf{U}_{n-1}\right\rangle \\
& =\left\langle\mathbf{Z}_{n}+\frac{1}{2}\left(\mathbf{Z}_{n}-\mathbf{Z}_{n-1}\right), \mathbf{Z}_{n}\right\rangle=\left|\mathbf{Z}_{n}\right|^{2}+\frac{1}{4}\left(\left|\mathbf{Z}_{n}\right|^{2}-\left|\mathbf{Z}_{n-1}\right|^{2}+\left|\mathbf{Z}_{n}-\mathbf{Z}_{n-1}\right|^{2}\right) .
\end{aligned}
$$

Hence (6.11) becomes

$$
\begin{aligned}
& \Delta t\left|\partial \mathbf{U}_{n}\right|^{2}+\frac{\Delta t^{3}}{4}\left|\partial^{2} \mathbf{U}_{n}\right|^{2}+\frac{\gamma}{2} \Delta t^{2}\left\|\partial \mathbf{U}_{n}\right\|^{2}+\hat{I}_{2}^{h}\left(\hat{\mathbf{U}}_{n}\right) \\
& \quad \leq \hat{I}_{2}^{h}\left(\hat{\mathbf{U}}_{n-1}\right)+\frac{C_{F} \Delta t^{2}}{2}\left|\partial \mathbf{U}_{n}\right|^{2} .
\end{aligned}
$$

$\mathbf{q}=3$. It is a calculation to show that

$$
\begin{aligned}
\Gamma_{3}= & \left\langle\mathbf{Z}_{n}+\frac{1}{2}\left(\mathbf{Z}_{n}-\mathbf{Z}_{n-1}\right)+\frac{1}{3}\left(\mathbf{Z}_{n}-2 \mathbf{Z}_{n-1}+\mathbf{Z}_{n-2}\right), \mathbf{Z}_{n}\right\rangle \\
= & \left|\mathbf{Z}_{n}\right|^{2}+\frac{1}{4}\left(\left|\mathbf{Z}_{n}\right|^{2}-\left|\mathbf{Z}_{n-1}\right|^{2}+\left|\mathbf{Z}_{n}-\mathbf{Z}_{n-1}\right|^{2}\right) \\
& +\frac{1}{3}\left[\frac{1}{2}\left(\left|\mathbf{Z}_{n}\right|^{2}-\left|\mathbf{Z}_{n-1}\right|^{2}+\left|\mathbf{Z}_{n}-\mathbf{Z}_{n-1}\right|^{2}\right)-\frac{1}{2}\left|\mathbf{Z}_{n}\right|^{2}\right. \\
& \left.\quad-\frac{1}{2}\left|\mathbf{Z}_{n-1}-\mathbf{Z}_{n-2}\right|^{2}+\frac{1}{2}\left|\mathbf{Z}_{n}+\mathbf{Z}_{n-2}-\mathbf{Z}_{n-1}\right|^{2}\right] \\
= & \frac{5}{6}\left|\mathbf{Z}_{n}\right|^{2}+\frac{5}{12}\left(\left|\mathbf{Z}_{n}\right|^{2}-\left|\mathbf{Z}_{n-1}\right|^{2}\right)+\frac{1}{4}\left|\mathbf{Z}_{n}-\mathbf{Z}_{n-1}\right|^{2} \\
& +\frac{1}{6}\left(\left|\mathbf{Z}_{n}-\mathbf{Z}_{n-1}\right|^{2}-\left|\mathbf{Z}_{n-1}-\mathbf{Z}_{n-2}\right|^{2}\right)+\frac{1}{6}\left|\mathbf{Z}_{n}+\mathbf{Z}_{n-2}-\mathbf{Z}_{n-1}\right|^{2}
\end{aligned}
$$

Hence (6.11) becomes

$$
\frac{5}{6} \Delta t\left|\partial \mathbf{U}_{n}\right|^{2}+\frac{\Delta t^{3}}{4}\left|\partial^{2} \mathbf{U}_{n}\right|^{2}+\frac{\Delta t}{6}\left|\partial \mathbf{U}_{n}-\Delta t \partial^{2} \mathbf{U}_{n-1}\right|^{2}+\frac{\gamma \Delta t^{2}}{2}\left\|\partial \mathbf{U}_{n}\right\|^{2}
$$




$$
\hat{I}_{3}\left(\hat{\mathbf{U}}_{n}\right) \leq \hat{I}_{3}\left(\hat{\mathbf{U}}_{n-1}\right)+\frac{C_{F} \Delta t^{2}}{2}\left|\partial \mathbf{U}_{n}\right|^{2}
$$

Setting $\tilde{\alpha}_{1}=\alpha_{1}=1, \tilde{\alpha}_{2}=\alpha_{2}=\frac{3}{2}$ and $\tilde{\alpha}_{3}=\frac{5}{3}$ we have the following proposition. THEOREM 6.2. Provided the timestep restriction

$$
\Delta t \leq \tilde{\alpha}_{q} / C_{F}
$$

holds then, for $q=1,2,3 \hat{I}_{q}(\bullet)$ is a Lyapunov function for the dynamical system $\hat{S}_{\Delta}^{h}$ on $\left\{\hat{L}_{2}^{h},\|\bullet\|\right\}$. Furthermore, $\hat{S}_{\Delta}^{h}$ is point dissipative on $\left\{\hat{L}_{2}^{h},\|\bullet\|\right\}$ and there exists a global attractor $\mathcal{A}^{h} \subset \hat{L}_{2}^{h}$ for the semigroup $\hat{S}_{\Delta}^{h}$.

Proof. The case $q=1$ is considered in Proposition 5.5. Clearly we just need to check condition I(iii) of $\S 2$ because I(ii) follows immediately from (6.12) and (6.13). We detail the case $q=3$ since the other case is treated in an identical fashion. It follows from (6.13) and the definition of $\hat{S}_{\Delta}^{h}$ that, if

$$
\hat{I}_{q}\left(\hat{S}_{\Delta}^{h}(\hat{\mathbf{V}})\right)=\hat{I}_{q}(\hat{\mathbf{V}})
$$

then, with $\hat{\mathbf{W}}=\hat{S}_{\Delta}^{h}(\hat{\mathbf{V}})$,

$$
\mathbf{W}^{(2)}=\mathbf{W}^{(3)}=\mathbf{V}^{(3)}=0 .
$$

Furthermore from (6.6) we have that $\mathbf{V}^{(2)}=0$ and $\mathbf{V}^{(1)}=\mathbf{W}^{(1)}$. Hence, by (6.5), it holds that

$$
A \mathbf{W}^{(1)}+M \mathbf{f}\left(\mathbf{W}^{(1)}\right)=0
$$

and $\hat{\mathbf{V}}=\hat{S}_{\Delta}^{h}(\hat{\mathbf{V}}) \in \hat{\mathcal{E}}^{h}$. By Lemma 2.1 the existence of a Lyapunov functional implies that $\hat{S}_{\Delta}^{h}$ is uniformly compact and hence that individual positive orbits are relatively compact; thus, by Proposition $2.4, \hat{S}_{\Delta}^{h}$ is point dissipative on $\left\{\hat{L}_{2}^{h},\|\bullet\|\right\}$. The existence of a global attractor follows from Proposition 2.5.

7. Numerical results. In this section we present numerical results which illustrate the material in $\S 3$. Numerical results illustrating the material in $\S \S 4$ and 5 may be found in [25].

We illustrate the spurious steady states and spurious stability properties implied by Theorem 3.2. For simplicity we assume, in addition to (F1) and (F4) on $f(u)$, that the equation is posed on the interval $\Omega=(0,1)$ and that $\operatorname{sgn}\left(f^{\prime \prime}(u)\right)=\operatorname{sgn}(u)$. (The canonical example is still $f(u)=u^{3}-u$.) It is proved in [3] that (1.1)-(1.3) has $2 n+1$ steady solutions for $\gamma \in\left[((n+1) \pi)^{-2},(n \pi)^{-2}\right)$. Thus the number of steady solutions approaches infinity as $\gamma \rightarrow 0_{+}$. Hence Theorem 2.1 does not seem to be worrying, at first glance - the method just resolves as many solutions as possible on the grid. However, a closer inspection shows that most of the numerical steady states are spurious: the true solutions have zeros which are equidistributed in the interval $[0,1]$ whereas most of the numerical steady states do not share this property. A rough calculation based on the number of zeros shows that, for $\gamma$ small, we would expect the numerical method to resolve approximately $2 J+1$ steady solutions (because of the equidistribuited zeros property). This implies the existence of approximately $3^{J}-2 J-1$ spurious solutions.

At this point it is important to understand the dynamics of the true equation, for $\gamma$ small. On a short time scale diffusion is negligible and the initial data rapidly 
(a)

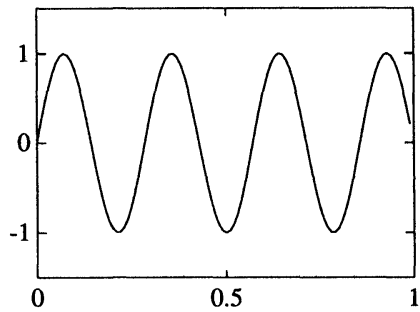

(c)

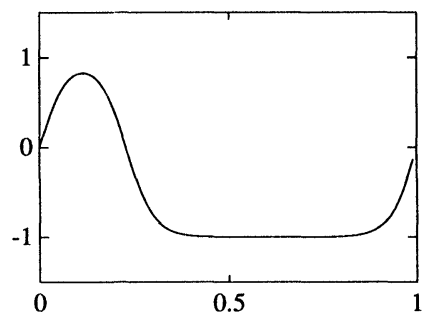

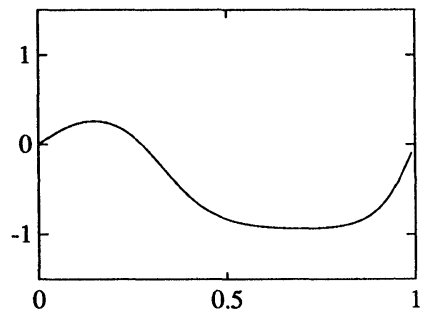

(b)

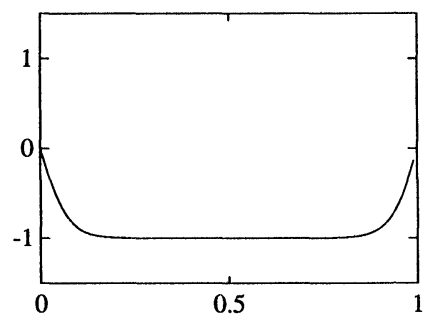

(d)

FIG. 7.1. $u(x, t)$ versus $x$ for solutions of (1.1)-(1.3). $\Omega=[0,1], \gamma=0.0025, u_{0}(x)=\sin (7 \pi x)$. For (a) $t=0$; (b) $t=20$; (c) $t=40$; (d) $t=80$. Implicit Euler applied to (1.1)-(1.3) with $\Delta t=0.1, \Delta x=0.01$.

evolves towards the stable zeros of $f, \pm 1$. Diffusion layers join together regions in which $u \approx 1$ and $u \approx-1$. Typically, these diffusion layers are not equidistributed and so they start to move towards a steady state configuration in which they are equidistributed. Furthermore, it is shown in [3] that, for $\gamma<\pi^{-2}$, only two of the steady states are stable and that these are one-signed in the interval $(0,1)$ - one negative and one positive. (Note that, in contrast, the numerical method has $2^{J}$ stable steady states.) The analysis of [2] shows that the evolution of interfaces occurs very slowly-on a time scale of $\mathcal{O}\left(\exp \left(-\gamma^{-\frac{1}{2}}\right)\right)$. The states which evolve on such a slow time scale are known as metastable states. Figures 7.1 and 7.2 show such an evolution starting from initial data $u_{0}(x)=\sin (7 \pi x)$. The first figure shows the solution at times $t=0,20,40$ and 80. Notice the devolopment of an interface seperating regions in which $u>0$ (to the left) and $u<0$ (to the right). The interface propagates to the boundary $x=0$ and, at $t=80$ the unique negative stable steady solution has been reached. The propagation is very slow and this can be seen in Fig. 7.2 where a three-dimensional projection of the solution $u(x, t)$ is shown.

The spurious steady states introduced by the discretisation are close in form to the metastable states; they are $\approx \pm 1$ everywhere except in transition layers. The resolution of the numerical method is insufficient to capture the tiny propagation speeds for the transition layers and hence these metastable states are stabilised by the discretisation and become steady solutions of the numerical method. Figures 7.3 and 7.4 show the numerical solution of (1.1)-(1.3) posed on the unit interval with the same initial data. In both cases the implicit Euler scheme is applied to (1.1)-(1.3). In Figure 7.3 a value of $\Delta x=0.05$ is taken and the solution approaches a spurious steady state. The computed solution is smooth and there is nothing obvious which tells us that it is spurious - it is only the fact that we know a priori that genuine steady solutions have equidistributed zeros that enables to rule out the computation as spurious. In Fig. 7.4 the value of $\Delta x$ is decreased to 0.01 . Notice that the computed solution now tends to a true steady solution; however, the density of the profiles indicates that the 


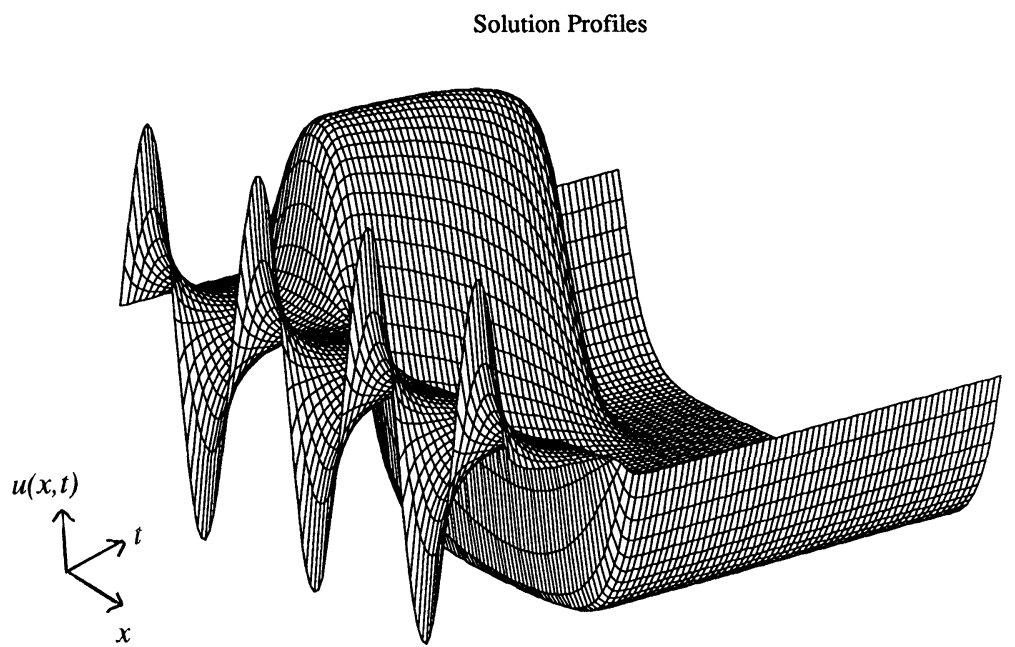

FIG. 7.2. Solution of (1.1)-(1.3). $\Omega=[0,1], \gamma=0.0025, u_{0}(x)=\sin (7 \pi x) .0 \leq t \leq 80$. Implicit Euler applied to (1.1)-(1.3) with $\Delta t=0.1, \Delta x=0.01$.

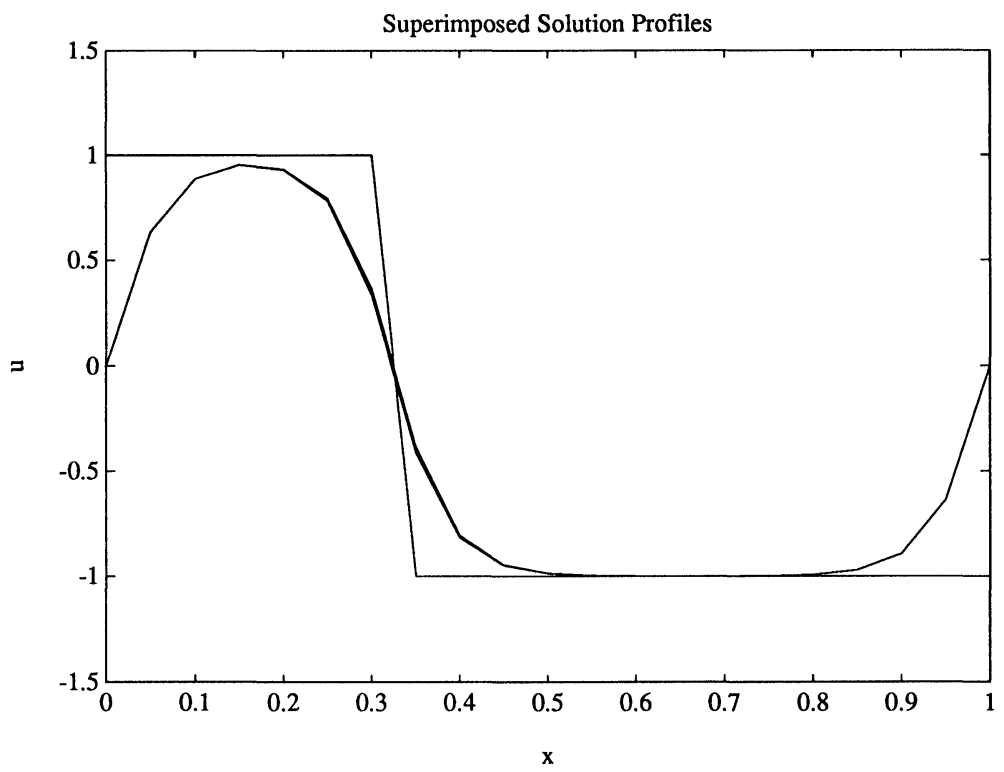

FIG. 7.3. $u(x, t)$ versus $x$ for solutions of (1.1)-(1.3). $\Omega=[0,1], \gamma=0.0025, u_{0}(x)=1, x<$ $0.3 ; u_{0}(x)=-1, x \geq 0.3$. Implicit Euler applied to (1.1)-(1.3) with $\Delta t=0.1, \Delta x=0.05$. Profiles at intervals of 10 seconds for $0 \leq t \leq 500$.

solution evolves to form a metastable state before finally approaching the negative stable steady state. The metastable state is clearly closely related to the spurious steady state in Fig. 7.3.

Figure 7.5 shows a trajectory approaching a steady solution which is unstable for the underlying PDE (since it changes sign) but which is artificially stabilised by the discretisation. To check that the solution is stable for the discretisation we took 


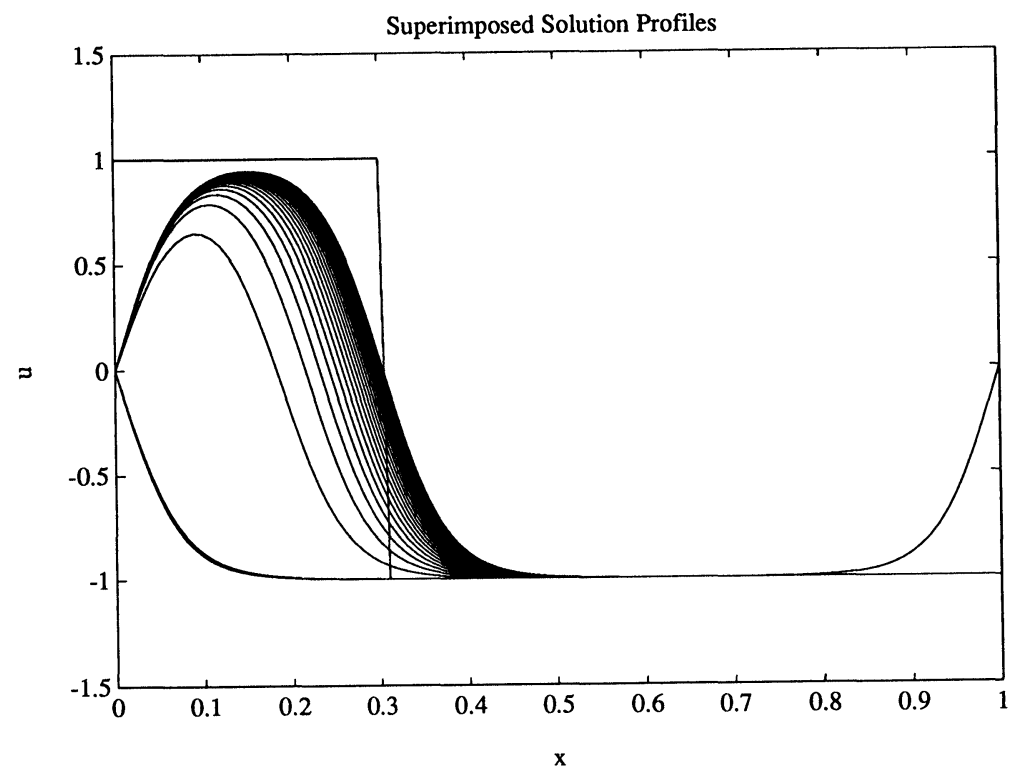

FIG. 7.4. Solutions of (1.1)-(1.3). $\Omega=[0,1], \gamma=0.0025, u_{0}(x)=1, x<0.3 ; u_{0}(x)=-1, x \geq$ 0.3. Implicit Euler applied to (1.1)-(1.3) with $\Delta t=0.1, \Delta x=0.01$. Profiles at intervals of 10 seconds for $0 \leq t \leq 500$.

the computed steady state from Fig. 7.5, denoted $u_{s}(x)$ in the caption of Fig. 7.6, and perturbed it, taking this as initial data. The results are shown in Fig. 7.6 which demonstrates the (spurious) stability of the steady solution.

Our numerical experiments are purely one dimensional but analogous properties hold in dimensions greater than one.

Appendix 1. Poincaré and inverse inequalities. Consider a diagonal matrix $M$ with positive diagonal entries and a positive definite symmetric matrix $A$ with eigenvectors and eigenvalues $\left\{\psi_{i}, \lambda_{i}\right\}$ satisfying

$$
A \psi_{i}=\lambda_{i} M \psi_{i}
$$

where

$$
C_{p}^{-2} \leq \lambda_{i} \leq C_{0} / h^{2}
$$

Under these assumptions the following inequalities hold for all $U \in \mathcal{R}^{J}$ :

$$
\begin{aligned}
|\mathbf{U}| & \leq C_{p}\|\mathbf{U}\|, \\
\|\mathbf{U}\|^{2} & \leq \frac{C_{0}}{h^{2}}|\mathbf{U}|^{2} \\
\|\mathbf{U}\| & \leq C_{p}\left|M^{-1} A \mathbf{U}\right|,
\end{aligned}
$$

and

$$
\left|M^{-1} A \mathbf{U}\right|^{2} \leq \frac{C_{0}}{h^{2}}\|\mathbf{U}\|^{2},
$$

where $C_{p}$ and $C_{0}$ are independent of $h$ and where $\langle\bullet, \bullet\rangle,|\bullet|$ and $\|\bullet\|$ are as defined in $\S 3$. 


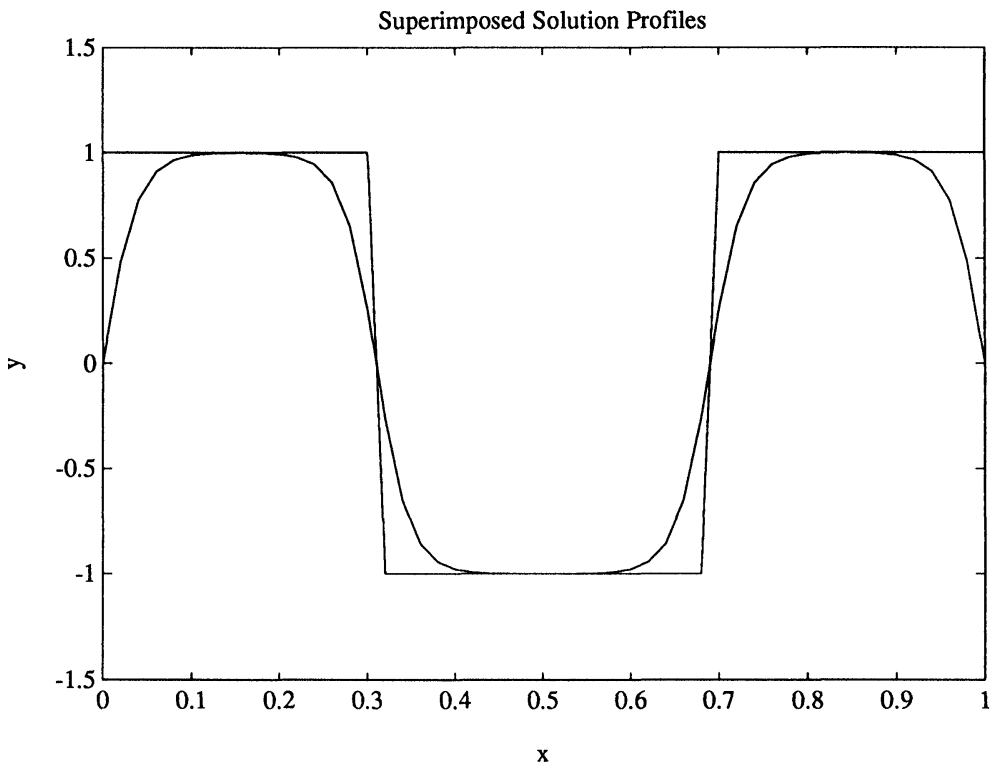

FIG. 7.5. $u(x, t)$ versus $x$ for solutions of $(1.1)-(1.3) . \quad \Omega=[0,1], \gamma=0.000784, u_{0}(x)=$ $1, x<0.3, x>0.7 ; \quad u_{0}(x)=-1,0.3 \leq x \leq 0.7$. Implicit Euler applied to (1.1)-(1.3) with $\Delta t=0.5, \Delta x=0.02$. Profiles at intervals of 50 seconds for $0 \leq t \leq 5000$.

Proof. Normalise the eigenvectors so that

$$
\left\langle\psi_{i}, \psi_{j}\right\rangle=\delta_{i j}
$$

Then and $\mathbf{U} \in \mathbb{R}^{J}$ may be written as

$$
\mathbf{U}=\sum_{i=1}^{J} a_{i} \psi_{i}
$$

and

$$
\begin{aligned}
A \mathbf{U} & =\sum_{i=1}^{J} a_{i} \lambda_{i} M \psi_{i} \\
|\mathbf{U}|^{2} & =\sum_{i=1}^{J} a_{i}^{2}
\end{aligned}
$$

and

$$
\|\mathbf{U}\|^{2}=\sum_{i=1}^{J} \lambda_{i} a_{i}^{2} \geq C_{p}^{-2} \sum_{i=1}^{J} a_{i}^{2} .
$$

Also,

$$
\|\mathbf{U}\|^{2} \leq \frac{C_{0}}{h^{2}} \sum_{i=1}^{J} a_{i}^{2}
$$




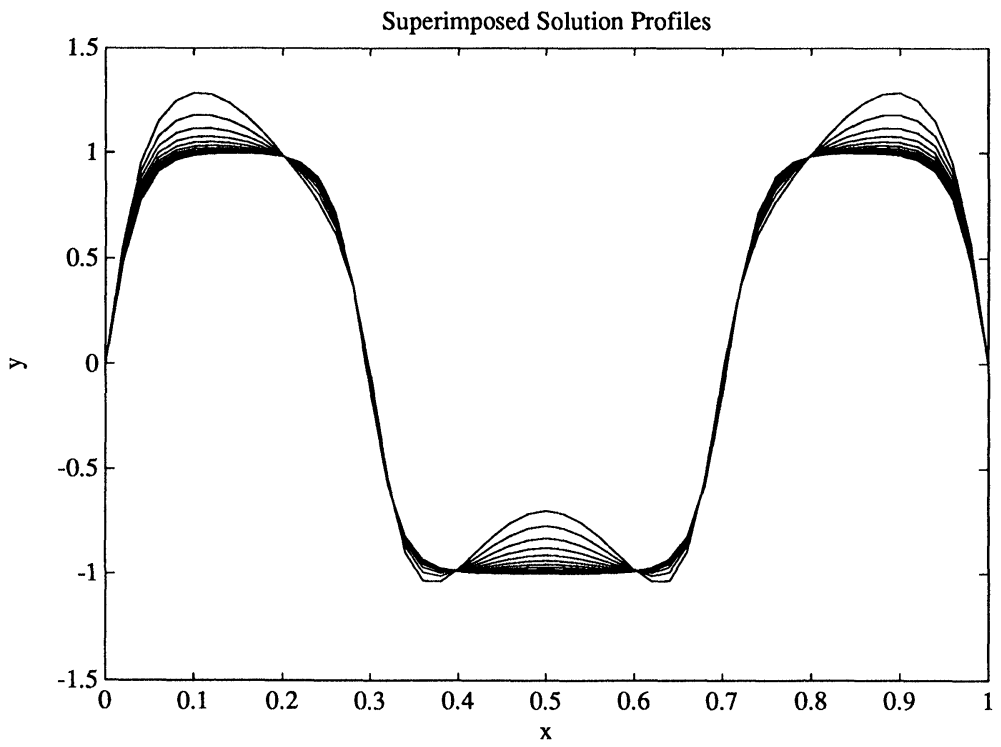

FIG. 7.6. $u(x, t)$ versus $x$ for solutions of (1.1)-(1.3). $\quad \Omega=[0,1], \gamma=0.000784, u_{0}(x)=$ $u_{s}(x)+0.2 * \sin (7 \pi x)$. Implicit Euler applied to $(1.1)-(1.3)$ with $\Delta t=0.2, \Delta x=0.02$. Profiles at intervals of 0.2 seconds for $0 \leq t \leq 10.0$

Hence the first pair of inequalities follow. Furthermore,

$$
M^{-1} A U=\sum_{i=1}^{J} a_{i} \lambda_{i} \psi_{i}
$$

and hence

$$
\left|M^{-1} A \mathbf{U}\right|^{2}=\sum_{i=1}^{J} a_{i}^{2} \lambda_{i}^{2}
$$

and the second pair of inequalities follow.

Appendix 2. A discrete uniform Gronwall lemma. Let $G_{n}, P_{n}, Y_{n}$ be three positive sequences satisfying, for all $n \geq n_{0}$ and $K_{0} \geq n_{0}$

$$
\begin{aligned}
\frac{Y_{n+1}-Y_{n}}{\Delta t_{n}} & \leq G_{n} Y_{n+1}+P_{n}, \quad \sum_{n=K_{0}}^{N+K_{0}} \Delta t_{n} G_{n} \leq a_{1}(r), \quad \sum_{n=K_{0}}^{N+K_{0}} \Delta t_{n} P_{n} \\
& \leq a_{2}(r), \quad \sum_{n=K_{0}}^{N+K_{0}} \Delta t_{n} Y_{n+1} \leq a_{3}(r),
\end{aligned}
$$

where

$$
\sum_{n=K_{0}}^{N+K_{0}} \Delta t_{n}=r
$$

If $\Delta t_{n} G_{n} \leq 1-\delta$ for all $n \geq n_{0}$, then

$$
Y_{N+K_{0}+1} \leq \exp \left(a_{1} / \delta\right)\left[\frac{a_{3}}{r}+a_{2}\right] \quad \forall K_{0} \geq n_{0}
$$


Proof. We have

$$
\left(1-\Delta t_{n} G_{n}\right) Y_{n+1}-Y_{n} \leq \Delta t_{n} P_{n}
$$

Let

$$
Q_{n+1}=\left(1-\Delta t_{n} G_{n}\right) Q_{n}, \quad n \geq n_{0}, \quad Q_{n_{0}}=1
$$

so that

$$
Q_{n+1} Y_{n+1}-Q_{n} Y_{n} \leq \Delta t_{n} P_{n} Q_{n}
$$

Summing we obtain

$$
Q_{N+K_{0}+1} Y_{N+K_{0}+1}-Q_{M} Y_{M} \leq \sum_{n=M}^{N+K_{0}} \Delta t_{n} P_{n} Q_{n} \leq Q_{M}\left(\sum_{n=M}^{N+K_{0}} \Delta t_{n} P_{n}\right)
$$

for $M \geq K_{0}$. Now

$$
Q_{N+K_{0}+1}=Q_{M} \Pi_{n=M}^{N+K_{0}}\left(1-\Delta t_{n} G_{n}\right)
$$

Hence

$$
Y_{N+K_{0}+1} \leq\left(Y_{M}+\sum_{n=M}^{N+K_{0}} \Delta t_{n} P_{n}\right) \Pi_{n=M}^{N+K_{0}}\left(1-\Delta t_{n} G_{n}\right)^{-1}
$$

Now

$$
(1-x)^{-1} \leq \exp (x /(1-x)), \quad|x|<1
$$

so that

$$
\left(1-\Delta t_{n} G_{n}\right)^{-1} \leq \exp \left(\Delta t_{n} G_{n} / \delta\right) .
$$

Hence

$$
\Pi_{n=M}^{N+K_{0}}\left(1-\Delta t_{n} G_{n}\right)^{-1} \leq \exp \left(\sum_{n=M}^{N+K_{0}} \Delta t_{n} G_{n} / \delta\right) \leq \exp \left(a_{1} / \delta\right) .
$$

Thus

$$
Y_{N+K_{0}+1} \leq\left(Y_{M}+a_{2}\right) \exp \left(a_{1} / \delta\right)
$$

Hence

$$
\begin{gathered}
\sum_{M=K_{0}+1}^{N+K_{0}+1} \Delta t_{M-1} Y_{N+K_{0}+1} \leq \exp \left(a_{1} / \delta\right)\left[\sum_{M=K_{0}+1}^{N+K_{0}+1} \Delta t_{M-1} Y_{M}+a_{2}\left(\sum_{M=K_{0}+1}^{N+K_{0}+1} \Delta t_{M-1}\right)\right] \\
\Rightarrow r\left(Y_{N+K_{0}+1}\right) \leq \exp \left(a_{1} / \delta\right)\left[a_{3}+a_{2} r\right]
\end{gathered}
$$

and division by $r$ gives the result. 
Appendix 3. Convergence of the number of discrete solutions. Throughout this appendix we use $C$ to denote a constant independent of $h$. By $\mathcal{G}$ we denote the Greens' operator for the negative Laplacian with homogeneous boundary data on $\Omega$ and we write the steady state equation for (1.1) as

$$
\gamma u=\mathcal{G} f(u) .
$$

Let $S^{h} \subset C(\bar{\Omega})$ denote the finite element space consisting of piecewise linear functions on an acute triangulation of $\Omega$ which vanish on $\partial \Omega$. The finite element approximation to (1.1) can be written as

$$
\gamma u^{h}=\mathcal{G}^{h} f\left(u^{h}\right),
$$

rather than the matrix version (3.8). Here $\mathcal{G}^{h} \in \mathcal{L}\left(C(\bar{\Omega}), S^{h}\right)$ is defined by

$$
\left\langle\nabla \mathcal{G}^{h} \eta, \nabla \xi\right\rangle=\langle\eta, \xi\rangle^{h}
$$

where $\langle\bullet, \bullet\rangle^{h}$ is the discrete $L^{2}$ inner product arising from lumped mass integration. It is known that (see [4])

$$
\left\|\left(\mathcal{G}-\mathcal{G}^{h}\right) \eta\right\| \leq C h\|\eta\|_{W^{1, q}(\Omega)}, \quad q \geq 2
$$

and

$$
\left\|\mathcal{G}^{h} \eta\right\|_{W^{1, p}(\Omega)} \leq c\left|I^{h} \eta\right|, \quad p \geq 1
$$

where $I^{h}$ is the interpolation operator from $C(\bar{\Omega})$ into $S^{h}$ and we use $\|\bullet\|$ and $|\bullet|$ for the $H_{0}^{1}(\Omega)$ and $L^{2}(\Omega)$ norms.

We suppose that (A1) has exactly $Q$ solutions $\left\{u_{i}\right\}$ for a given $\gamma$ and that there exists $\delta>0$ such that in the balls

$$
B_{i}^{\delta}=\left\{\eta:\left\|\eta-u_{i}\right\| \leq \delta\right\}
$$

there is only one solution of (A1). Thus, in the closed set

$$
V^{\delta}=H_{0}^{1}(\Omega) \backslash\left(\bigcup_{i} B_{i}^{\delta}\right)
$$

there are no solutions and

$$
\varepsilon=\inf _{\eta \in V^{\delta}}\|\gamma \eta-\mathcal{G} f(\eta)\|>0
$$

Hence, if $u^{h} \in V^{\delta}$ then

$$
\begin{aligned}
\varepsilon & \leq\left\|\gamma u^{h}-\mathcal{G} f\left(u^{h}\right)\right\|=\left\|\gamma u^{h}-\mathcal{G}^{h} f\left(u^{h}\right)+\left(\mathcal{G}^{h}-\mathcal{G}\right) f\left(u^{h}\right)\right\| \\
& \leq\left\|\left(\mathcal{G}^{h}-\mathcal{G}\right) f\left(u^{h}\right)\right\| \leq C h\left\|f\left(u^{h}\right)\right\|_{W^{1, q}(\Omega)} .
\end{aligned}
$$

Using the $L^{\infty}$ and $H_{0}^{1}$ bounds on $u^{h}$ from Lemma 3.1 we find that

$$
\left\|f\left(u^{h}\right)\right\|_{W^{1, q}(\Omega)} \leq C
$$

and so $u^{h} \in V^{\delta}$ implies that $\varepsilon \leq C h$. Thus for $h<h_{0}=\varepsilon / C$ we have that $u^{h}$ belongs to one of the balls $B_{i}^{\delta}$.

If $\left(\gamma, u_{j}\right)$ is a regular point of (A1) then the analysis in [4, Chap. 3] implies that for $h$ sufficiently small there is a unique solution $u^{h}$ in the ball $B_{j}^{\delta}$. 
Acknowledgments. A preliminary version of this work was presented at the IMA (UK) "Dynamics of Numerics and Numerics of Dynamics" conference, July 1990, Bristol. The work of C. M. Elliott was partially supported by the Institute of Mathematics and its Applications (Minneapolis) with funds provided by the National Science Foundation during the 1990/91 program on "Phase Transitions and Free Boundaries." The authors are grateful to Arieh Iserles for helpful discussions regarding the material in $§ 3.1$.

\section{REFERENCES}

[1] J. Bence, B. Merriman, AND S. Osher, On spurious numerical solutions of fast reaction, slow diffusion equations, in preparation.

[2] J. CARR AND R. Pego, Metastable patterns in solutions of $u_{t}=\varepsilon^{2} u_{x x}-f(u)$, Comm. Pure Appl. Math., 42 (1989).

[3] N. ChAFEe AND E. INFANTE, $A$ bifurcation problem for a nonlinear parabolic problem, J. Appl. Anal., 4 (1974), pp. 17-37.

[4] M. Crouzeix AND J. Rappaz, On Numerical Approximation in Bifurcation Theory, MassonSpringer-Verlag, Paris, 1990.

[5] C. M. ElliotT, The Cahn-Hilliard model for the kinetics of phase separation, in Mathematical Models for Phase Change Problems, J. F. Rodrigues, ed., Birkhauser-Verlag, Basel, Switzerland, 1989.

[6] C. Foias, M. S. Jolly, I. G. Kevrekidies, and E. S. Titi, Dissipativity of numerical schemes, Nonlinearity, 4 (1991), pp. 591-613.

[7] D. F. GRIFFiths AND A. R. Mitchell, Stable periodic bifurcations of an explicit discretisation of a nonlinear partial differential equation in reaction diffusion. IMA J. Numer. Anal., 8 (1988), pp. 435-454.

[8] J. HALE, Asymptotic behaviour of dissipative systems, AMS Mathematical Surveys and Monographs 25, American Mathematical Society, Providence, RI, 1988.

[9] J. HALE, X.-B. LIN, AND G. RAUGEL, Upper semicontinuity of attractors for approximations of semigroups and partial differential equations, Math. Comp., 50 (1988), pp. 89-123.

[10] J. HALE AND G. RAUGEL, Lower semicontinuity of attractors of gradient systems and applications, Ann. Mat. Pura ed Applicata, 4 (1989), pp. 281-326.

[11] D. Henry Geometric Theory of Semilinear Parabolic Equations, Lecture Notes in Math. 840, Springer-Verlag, New York, 1981.

[12] D. HofF, Stability and convergence of finite difference methods for systems of nonlinear reaction-diffusion equations, SIAM J. Numer. Anal., 15 (1978), pp. 1161-1177.

[13] A. R. Humphries, Spurious solutions of numerical methods for initial value problems, IMA J. Numer. Anal., to appear.

[14] A. R. Humphries AND A. M. StUART, Runge-Kutta methods for dissipative and gradient dynamical systems, SIAM J. Numer. Anal., to appear.

[15] A. IsERLES, private communication, 1990.

[16] P. E. KLOEDEN AND J. LORENZ, Lyapunov stability and attractors under discretisation, Proceedings EQUADIFF '87, C. M. Dafermos et al., eds., Marcel-Dekker, New York, 1989.

[17] S. N. S. KHALSA, Finite element approximation of a reaction diffusion equation. Part I: application of a topological technique to the analysis of asymptotic behaviour of the semidiscrete equation, Quart. Appl. Math., 44 (1986), pp. 375-386.

[18] J. D. Lambert, Numerical Methods for Ordinary Differential Equations, Wiley, London, 1991.

[19] H. MATANo, Asymptotic behaviour and stability of solutions of semilinear diffusion equations, Publ. RIMS, Kyoto Univ., Kyoto, Japan, 15 (1979), pp. 401-454.

[20] R. Nochetto, Finite element methods for parabolic free boundary problems, in Advances in Numerical Analysis, Vol. 1, W. Light, ed., Oxford University Press, Cambridge, 1991.

[21] R. D. Richtmyer AND K. W. Morton, Difference Methods for Initial Value Problems, Academic Press, New York, 1967.

[22] J. SHEN, Convergence of approximate attractors for a fully discrete system for reactiondiffusion equations, Numer. Funct. Anal. Optim., 10 (1989), pp. 1213-1234.

[23] A. B. STEPhens AND G. R. ShUBin, Multiple solutions and bifurcations of finite difference approximations to some steady problems of fluid mechanics, SIAM J. Sci. Statist. Comput., 2 (1981), pp. 404-415.

[24] A. M. STUART, Linear instability implies spurious periodic solutions, IMA J. Numer. Anal., 9 (1989), pp. 465-486. 
[25] A. M. Stuart and A. T. Peplow, The dynamics of the theta method, SiAM J. Sci. Statist. Comput., 12 (1991), pp. 1351-1372.

[26] R. Temam, Infinite Dimensional Dynamical Systems in Mechanics and Physics, SpringerVerlag, New York, 1988.

[27] T. J. ZELENYAK, Stabilization of solutions of boundary value problems for a second order parabolic equation with one space variable, Differential Equations, 4 (1968), pp. 17-22.

[28] J. SMOLLER, Shock waves and reaction-diffusion equations, Springer-Verlag, New York, 1983. 